\title{
New Records for The Turkish Freshwater Algal Flora in Twenty-five River Basins of Turkey, Part II: Chlorophyta, Cyanobacteria, Euglenozoa
}

\author{
Faruk Maraşlıoğlu ${ }^{1, *}(\mathbb{D})$, Burak Öterler ${ }^{2}(\mathbb{D})$, Tuğba Ongun Sevindik ${ }^{3}\left({ }^{(}\right)$, Elif Neyran Soylu ${ }^{4}(\mathbb{D}$, \\ Nilsun Demir $^{5}{ }^{(D}$, Abuzer Çelekli6 ${ }^{(D}$, Haşim Sömek ${ }^{7}{ }^{(0)}$, Tolga Coşkun ${ }^{5}$, Cüneyt Nadir

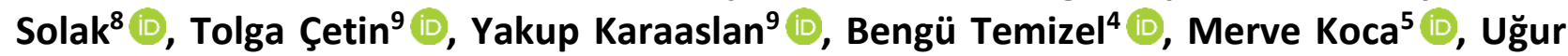

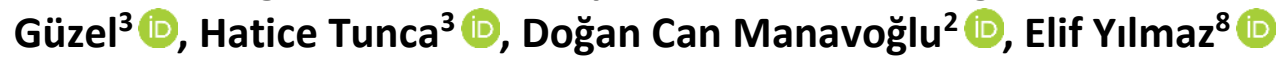

\author{
${ }^{1}$ Hitit University, Faculty of Arts and Science, Department of Biology, 19040 Çorum, Turkey. \\ ${ }^{2}$ Trakya University, Faculty of Science, Department of Biology, 22030 Edirne, Turkey. \\ ${ }^{3}$ Sakarya University, Faculty of Arts and Science, Department of Biology, 54187 Adapazarı, Turkey. \\ ${ }^{4}$ Giresun University, Faculty of Arts and Science, Department of Biology, 28100 Giresun, Turkey. \\ ${ }^{5}$ Ankara University, Faculty of Agriculture, Department of Fisheries and Aquaculture Engineering, 06110 Ankara, Turkey. \\ ${ }^{6}$ Gaziantep University, Faculty of Art and Science, Department of Biology, 27310 Gaziantep, Turkey. \\ ${ }^{7}$ İzmir Katip Çelebi University, Faculty of Fisheries, Department of Aquaculture, İzmir, Turkey. \\ ${ }^{8}$ Dumlupınar University, Faculty of Arts and Science, Department of Biology, Kütahya, Turkey. \\ ${ }^{9}$ T.R. Ministry of Agriculture and Forestry, Directorate General of Water Management, Ankara, Turkey.
}

\section{How to cite}

Maraşlıoğlu, F., Öterler, B., Ongun Sevindik, T., Soylu, E.N., Demir, N., Çelekli, A., Sömek, H., Coşkun, T., Solak, C.N., Çetin, T., Karaaslan, Y., Temizel, B., Koca, M., Güzel, U., Tunca, H., Manavoğlu, D.C., Yılmaz, E. (2022). New Records for The Turkish Freshwater Algal Flora in Twenty-five River Basins of Turkey, Part II: Chlorophyta, Cyanobacteria, Euglenozoa. Turkish Journal of Fisheries and Aquatic Sciences, 22(8), TRJFAS19319. http://doi.org/10.4194/TRJFAS19319

\section{Article History}

Received 23 February 2021

Accepted 03 February 2022

First Online 09 February 2022

\section{Corresponding Author}

Tel.: +903642230800

E-mail: farukmaraslioglu@hitit.edu.tr

\section{Keywords}

Phytoplankton

Taxonomy

First record

Lakes

Turkey

\section{Introduction}

It is thought that due to their small size, high abundance, fast population growth, and long-range dispersal, algae such as other microorganisms are considered as cosmopolitan because they occur in very diverse habitats and distribute globally (Fenchel and Finlay, 2004; Hillebrand, 2004; Graneli and Turner, 2006; Reynolds, 2006). However, recent studies reported that spatial diversity patterns also exist for algae (Ptacnik et al., 2010; Stomp et al., 2011; Wang et al., 2011; Maileht et al., 2013). Some dispersal agents such as a river, air, animals, human are needed for dispersal of algae (Padisák et al., 2016), however, many studies indicated the role of several factors, such as productivity, nutrient and light availability, alkalinity, periodic disturbances, food web structure, colonization processes, dispersal ability among local communities (Tilman et al., 1982; Sommer, 1993; Ricklefs, 1987; Leibold, 1996; Morin and Fox, 2004; Smith et al., 2005; Stomp et al., 2007) for the spatial diversity of algae. Geographical variation (latitudinal, longitudinal, and altitudinal gradients) and lake morphometry have been shown to control these factors and are considered as the major determinant of 
phytoplankton diversity (Stomp et al., 2011; Winslow et al., 2015; Borics et al., 2016). Due to the effects of three different types of climate, geographical variation, and different soil types, 23 lake typologies were determined in Turkish lakes (DGWM, 2015a), and these lakes have great potential to support distinct algal diversity.

Although several studies were recorded on phytoplankton composition, diversity, and abundance (Maraşlıoğlu et al., 2005; Sömek et al., 2005; Demir et al., 2014; Sevindik et al., 2017a, Öterler et al., 2018; Çelekli et al., 2020), in recent years, several projects have also been implemented and funded by the Ministry of Agriculture and Forestry, Directorate General of Water Management (DGWM), and General Directorate of State Hydraulic Works (DSi). This study is also a part of the "Establishment of Reference Monitoring Network in Turkey" project which is supported by DGWM. In this project, 275 lakes in 25 river basins were studied, and a total of 1363 phytoplankton taxa were detected. Among these taxa, 330 Chlorophyta, 164 Cyanobacteria, and 187 Euglenozoa taxa were determined.

A few check-lists were published (Gönülol et al., 1996; Aysel, 2005; Şahin, 2005), and many new records were given for the algal flora of Turkey (Aysel et al., 1993; Öztürk et al., 1995a, 1995b; Şahin 1998, 2000, 2002, 2007, 2009; Şahin and Akar, 2007; Apaydın-Yağcı and Turna, 2002; Atıcı, 2002; Baykal et al., 2009, 2012; Sevindik et al. 2010, 2011, 2015, 2017b; Özer et al., 2012; Akar and Şahin, 2014; Yüce and Ertan, 2014; Varol and Fucikova, 2015; Varol and Şen, 2016; Morkoyunlu and Aktaş, 2020). Therefore, the total number of taxa has increased (Taşkın et al., 2019; Maraşlıoğlu and Gönülol, 2021). Although 6717 Chlorophyta, 4788 Cyanobacteria, and 1513 Euglenozoa taxa were reported in previous studies in the world (Guiry and Guiry, 2021), only 1541 taxa belonging to these three divisions have been listed in Turkey so far (Maraşlıoğlu and Gönülol, 2021).

This study is one of the outcomes of the "Establishment of Reference Monitoring Network in Turkey" project, financially and technically supported by DGWM. In this project, 275 lakes in 25 river basins were studied, and a total of 1363 phytoplankton taxa were detected. It is thought that a total of 238 new records belonging to three divisions (Chlorophyta, Cyanobacteria, Euglenozoa) whose figures and habitats were given in this study will contribute to the studies on freshwater algal flora of Turkey.

\section{Materials and Methods}

\section{Study Area}

Turkey has 25 river basins (Figure 1), and inland water bodies in these basins consist of 200 natural lakes, 806 reservoirs, and 1000 ponds. Considering the areas of river basins, the annual amount of water produced per unit area is lowest in Akarçay Basin with 64.430 $\mathrm{m}^{3} / \mathrm{km}^{2}$, while highest in Eastern Karadeniz Basin with
$618.850 \mathrm{~m}^{3} / \mathrm{km}^{2}$ (Foreign Relation Office of DSi, 2014). However, Lakes Region (Burdur Basin), South Marmara (Susurluk Basin), Lake Van and its surroundings (Lake Van Basin), Lake Tuz, and its surroundings (Konya Basin) were the regions where the natural lakes are gathered (Hoşgören, 1994).

A total of 275 lakes, including reservoirs, were sampled during the study in 25 river basins. The number of studied lakes in the river basins was given in Table 1. These lakes are grouped in 22 lake typologies based on altitude (R), lake depth (D), lake size (A), and geology (J) (DGWM, 2015a), and they are located between the longitudes of $26^{\circ} 19^{\prime}$ and $43^{\circ} 54^{\prime} \mathrm{E}$ and the latitudes of $35^{\circ} 56^{\prime}$ and $42^{\circ} 00^{\prime} \mathrm{N}$. The altitudes of the sampled lakes vary between sea level (Lake Gala) and 2757 m (Lake Çamlu).

\section{Sampling and Identification}

Phytoplankton was sampled three times (spring, summer, and fall) a year during 2017 and 2019 at the one, two, or three monitoring stations in each lake. Station numbers were determined as one for lakes that have a surface area smaller than 50 ha, two for lakes that have a surface area between 50 and 500 ha and, three for lakes that have a surface area higher than 500 ha (DGWM, 2015b). One of the selected stations was determined at the deepest point of the lake. Three depths (surface, middle, and bottom) of the euphotic depth (Secchi disk depth $\times 2.5$ ) were sampled with a Ruttner water sampler (Hydro-Bios $2 \mathrm{~L}, 50 \mathrm{~cm}$ long) in the lakes, then a subsample was taken from mixed water of the three depths. Plankton net (Hydro-Bios 438001, $50 \times 25 \mathrm{~cm}$ ) with a pore diameter of $50 \mu \mathrm{m}$ was also used for sampling. Samples were fixed with Lugol's solution. After field sampling, the samples were taken to the laboratories of different universities (Sakarya University, Hitit University, Giresun University, Ankara University, Gaziantep University, İzmir Katip Çelebi University, Trakya University). In these laboratories, phytoplankton samples were firstly prepared for identification and then, phytoplankton enumerations were carried out according to the standard method (Anonymous, 2006). Both compound and inverted microscopes (Olympus BX53, Olympus CKX41, Olympus BX53F (DIC), Olympus BX51, Olympus IX81, Nikon Eclipse Ts2, Leica DM750) were used, since new species were detected both in the identification and enumeration processes according to the literature (Heering, 1914; Geitler, 1925; Desikachary, 1959; Huber-Pestalozzi, 1961, 1962, 1969, 1972, 1983; Philipose, 1967; Compère, 1986; Ettl and Gärtner, 1988; John et al., 2003; Komárek and Anagnostidis, 1999; Dillard, 2000; Komárek and Anagnostidis, 2005; Joosten, 2006; Baker and Fabbro, 2002; Park, 2012; Komárek, 2013). Identified taxa were checked with the checklist of Aysel (2005), Taşkın et al. (2019), and the database of Turkish algae (Maraşlıoğlu and Gönülol, 2021), and then determined as new taxa for Turkish freshwater algal flora. The currently 


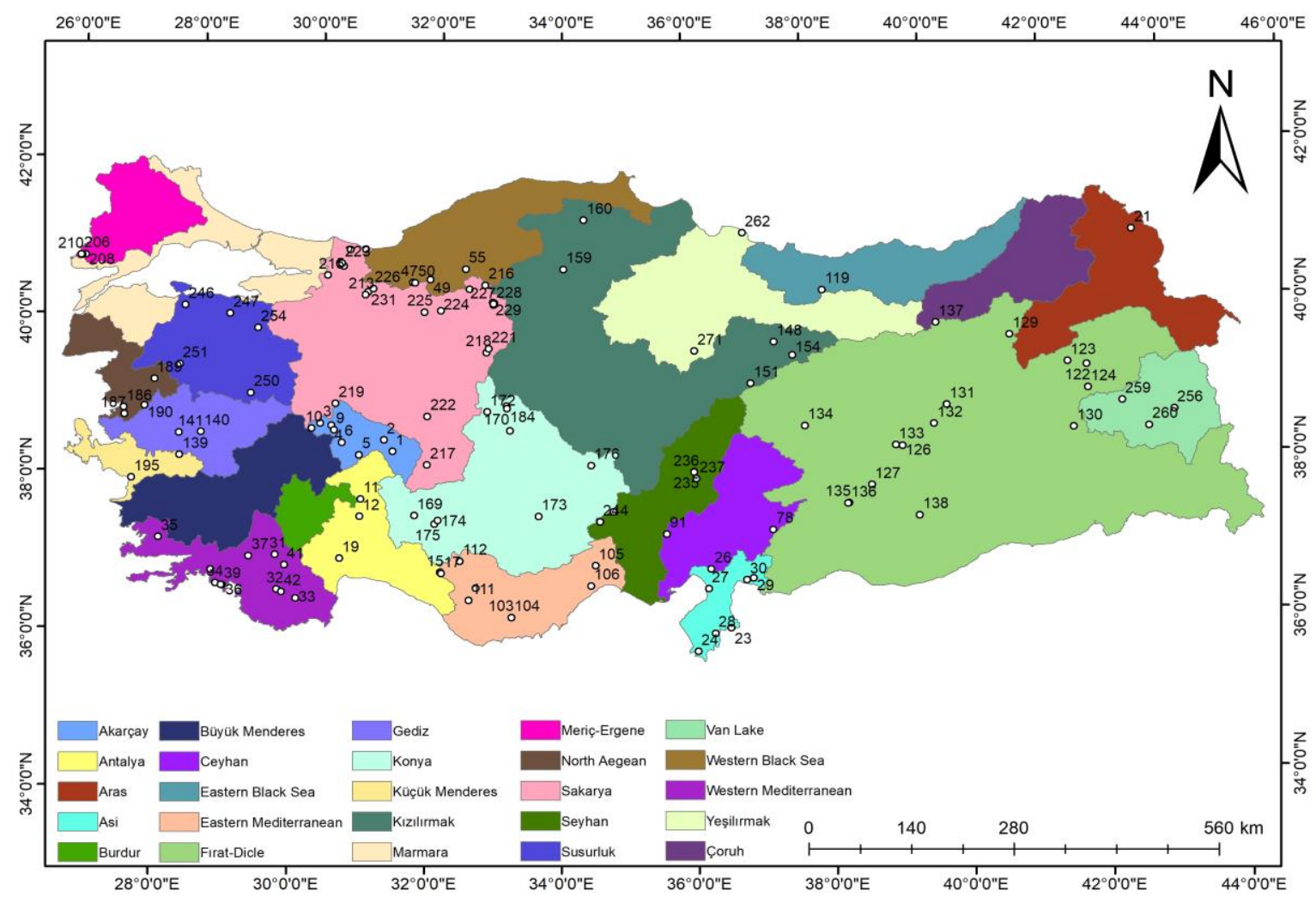

Figure 1. Map of sampling locations and river basins in Turkey.

Akarçay Basin; (1) Akşehir Lake, (2) Eber Lake, (3) Akdeğirmen Reservoir, (4) 26 Ağustos TP Lake, (5) Karamık Reeds, (6) Ağzıkara Pond, (9) Şehit Uz. Çvş. Nurullah Oymak Pond, (10) Tazlar Satı Gelin Pond, Antalya Basin; (11) Eğirdir Lake, (12) Kovada Lake, (15) Duruca Lake, (17) Küllü Lake, (19) Düden Lake, Aras Basin; (21) Çıldır Lake, Asi Basin; (23) Reyhanlı (Yenihisar) Lake, (24) Yayladağ Reservoir, (26) Karagöl Lake, (27) Adsız Lake, (28) Yarseli Reservoir, (29) Üçpınar Pond, (30) Sapkanlı Pond, Western Mediterranean Basin; 31) Gölhisar Lake, (32) Girdev Lake, (33) Avlan Lake, (34) Dalaman Wetlands, (35) Denizcik Lake, (36) Kocagöl Lake, (37) Kusuru Lake, (38) Köyceğiz Lake, (39) Küçükdalyan Lake, (41) Yazır Lake, (42) Baranda Lake, Western Black Sea; (47) Parçayır Lake, (49) Dipsiz Lake, (50) Gölcük Lake, (55) Koca Lake, Ceyhan Basin; (78) Kartalkaya Reservoir, (91) Zerdali Pond, Çoruh Basin; (98) Şavşat Karagöl Lake, Eastern Mediterranean Basin; (103) Aygır Lake, (104) Uzun Lake, (105) Değirmendere Pond, (106) Cemilli Çevlik Pond, (110) Başyayla Pond, (111) Göktepe Pond, (112) Bağbaşı Reservoir, Eastern Black Sea Basin; (119) Çamlu Lake, Fırat-Dicle Basin; (122) Kaz Lake, (123) Ahır Lake, (124) Haçlı Lake, (126) Hazar Lake, (127) Karagöl Lake, (129) Palandöken Pond, (130) Güroymak Reservoir, (131) Kalecik Reservoir, (132) Kapraçmaz Pond, (133) Dedeyolu Pond, (134) Güzelyurt Sulama Pond, (135) Hasancık Pond, (136) Incesu Pond, (137) Otlukbeli Lake, (138) Siverek Yeleken Pond, Gediz Basin; (139) Gölcük Lake, (140) Demirköprü Reservoir, (141) Marmara Lake, Kızılırmak Basin; (148) Hafik Lake, (151) Arı Lake, (154) Dipsiz Lake, Lake-1, (159) Yeşilgöl 1 Lake, (160) Bardakçılı Mevki Lake, Konya Basin; (169) Beyşehir Lake, (170) Tuz Lake, (172) Gök (Kozanlı) Lake, (173) Meke Lake (Meke Maarı), (174) Gavur Lake, (175) Dipsiz Lake, (176) Acıgöl Lake 2, (181) Düden Lake, (184) Küçük Lake, North Aegean Basin; (186) Boz Lake, (187) Güzelhisar Reservoir, (189) Sevişler Reservoir, (190) Tepe Lake, Küçük Menderes Basin; (195) Gebekirse Lake, Meriç-Ergene Basin; (206) Gala Lake, (208) Pamuklu Lake, (210) Domuz Lake, Sakarya Basin; (211) Taşkısığı Lake, (212) Akgöl 2 Lake, (213) Çubuk Lake, (214) Poyrazlar Lake, (215) Sapanca Lake, (216) Işık Dağı Karagöl Lake, (217) Çavuşcu Lake, (218) Mogan Lake, (219) Üçlerkayası Pond, (220) Çubuk Karagöl Lake, (221) Eymir Lake, (222) Akgöl 1 Lake, (223) Küçük Akgöl Lake, (224) Avdan Lake, (225) Kayuslu Lake, (226) Karamurat Lake, (227) Cüneyt Sönmez Pond, (228) Çılgınlar Pond, (229) Yıldırım Evci Pond, (231) Sülüklü Lake, (232) Çamkoru TP Pond, (233) Anagöl Lake, Seyhan Basin; (235) Tufanbeyli Demiroluk Pond, (236) Adsız Lake, (237) Pekmezli-Çatalçam Pond, (243) Topacık Pond, (244) Hüsniye Pond, Susurluk Basin; (246) Manyas Lake, (247) Uluabat Lake, (250) Gölcük Lake, (251) ikizcetepeler Reservoir, (254) Nilüfer Reservoir, Van Lake Basin; (256) Erçek Lake, (259) Aygır Lake, (260) Van Lake, Yeşilırmak Basin; (262) Akgöl Lake, (271) Dipsiz Lake 2

Note:*Maraşlıŏlu et al. (2021)

Table 1. Number of studied lakes in 25 river basins

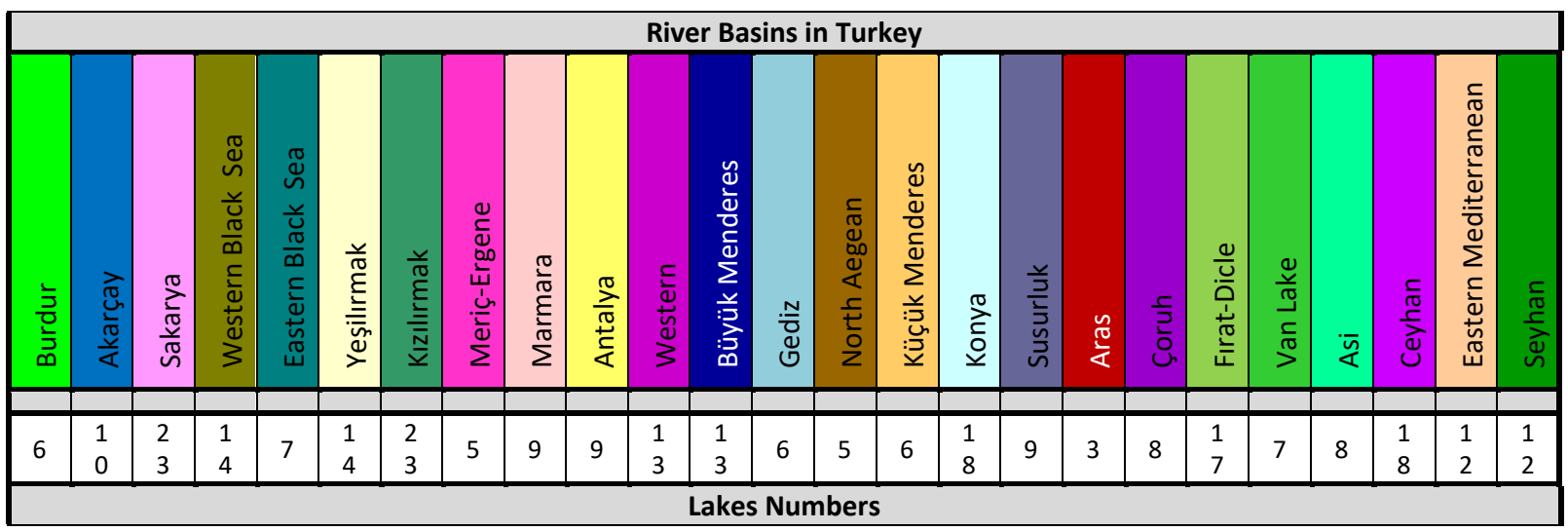


accepted nomenclature and distribution of taxa have been given according to Guiry and Guiry (2021). The author names were abbreviated according to Brummitt and Powell (1992). Taxa were photographed with imaging systems (LAS v.4.8 program, CellSens Vers. 1.6 program) and a camera (Leica MFC170 HD model, DP73 model, Olympus SC100 model) attached to various microscopes.

\section{Results}

A total of 330 Chlorophyta, 187 Euglenozoa, and 164 Cyanobacteria taxa were determined in a study conducted from 2017 to 2019 in 25 river basins of Turkey. 116 Chlorophyta, 81 Euglenozoa, and 41 Cynabacteria taxa were identified as new records for the freshwater algae of Turkey during our study. General information and figures of these taxa were presented in Table 2 and Figure 2-23.

New records were found in 124 of 275 lakes from 25 basins of Turkey. The first three basins with the highest number of lakes recorded new species were Sakarya (22 lakes), Firat-Dicle (15 lakes) and Western Mediterranean (11 lakes) basins, respectively. The basins with no new records were Burdur, Büyük Menderes and Marmara basins. Western Mediterranean (43 taxa), Firat-Dicle (41 taxa) and Asi (38 taxa) basins constitute $55.9 \%$ of the total new records. The first five lakes with the highest number of new recorded taxa were respectively Girdev Lake (22 taxa) from Western Mediterranean basin, Cüneyt Sönmez Pond (18 taxa) from Sakarya basin, Yarseli Reservoir (16 taxa) from Asi basin, Siverek Yeleken Pond (16 taxa) from Fırat-Dicle basin, and Üçlerkayası Pond (15 taxa) from Sakarya basin.

\section{Discussion}

This study reports 238 phytoplankton taxa as new records for the freshwater algal flora of Turkey from the 25 river basins. The diversity of new records presented in this study includes 41 taxa from Cyanobacteria, 81 taxa from Euglenozoa, and 116 taxa from Chlorophyta divisions. The highest genus diversity was seen in Chlorophyta with 57 genera, while the lowest genus diversity was seen in Euglenozoa with 12 genera. Also, 28 genera were identified in Cyanobacteria division.

While $75.6 \%$ of 41 new records from Cyanobacteria detected in 25 river basins in Turkey are rare species as the distribution area, $24.4 \%$ of them are the species that are widely seen in the world. Although Anabaena laxa, Anathece minutissima, A. smithii, Aphanocapsa conferta, Dolichospermum lemmermannii, Microcystis botrys, Nostoc caeruleum, Nodularia sphaerocarpa, Oscillatoria trichoides, and Rhabdoderma lineare species are commonly reported, Anabaena oblonga, $A$. sphaerica var. attenuata, Anabaenopsis milleri, Aphanizomenon klebahnii, Aphanocapsa nubila, Arthrospira platensis var. non-constricta, Aphanothece atrocrustacea, A. comasii, A. floccosa, Chroococcus lithophilus, C. mipitanensis, Coelosphaerium aerugineum, Gloeothece subtilis, Leptolyngbya ectocarpi, Limnospira fusiformis, Oscillatoria chlorina f. perchlorina, Pannus spumosus, Phormidium schultzii Planktolyngbya microspira, Planktothrix isothrix, Pulvinularia suecica, Romeria gracilis, Snowella litoralis, S. fennica, Synechocystis parvula, Woronichinia botrys, and $W$. karelica taxa are rarely reported in worldwide. Also, no distributional records were found in algaebase about the distribution areas of Aphanocapsa planctonica, Merismopedia hyalina, Anabaenopsis rippkae, and Dolichospermum fallax species (Guiry and Guiry, 2021). Among the new record species in Cyanobacteria, the ones with common distribution are generally seen in the Sakarya Basin, while those with rare distribution areas were mostly detected in FiratDicle and Western Mediterranean basins.

A great majority of 81 new records from Euglenozoa detected in 25 river basins in Turkey are rare species in the world. However, Euglena velata, Euglenaria anabaena, Phacus carinatus, and $P$. caudatus are common species in the world (Guiry and Guiry, 2021). Most of the new records detected from Euglenozoa group were tolerant taxa as water quality indicators (Phillips et al., 2010). Among the new records in Euglenozoa, species with rare distribution areas were mostly found in Sakarya and Firat-Dicle basins, while such a predominant basin could not be detected for common species.

While $78,4 \%$ of 116 new records from Chlorophyta detected in 25 basins in Turkey are rare species, $21.6 \%$ of them are the species that are widely seen in the world. Acanthosphaera zachariasii, Ankistrodesmus stipitatus, Aulacomonas submarina, Characium angustum, Chlamydomonas anglica, C. nivalis, Chlorella chlorelloides, Chlorolobion lunulatum, Desmodesmus abundans var. brevicauda, Dicellula geminata, Dictyosphaerium subsolitarium, Didymocystis inermis, Didymogenes palatina, Kirchneriella major, Monoraphidium obtusum, M. pseudobraunii, Planktococcomyxa lacustris, Palmococcus reniformis, Paulschulzia pseudovolvox, Pediastrum ovatum, $P$. simplex var. clathratum, Pseudopediastrum subgranulatum, Quadrigula chodatii, Q. sabulosa, and Willea crucifera taxa are commonly distributed in the word. However, the remaining 91 new records from Chlorophyta were identified as rare taxa (Guiry and Guiry, 2021). In this study, among the new record species from Chlorophyta, the Sakarya Basin was the main area where taxa with both common and rare distribution areas were most frequently found. In Chlorophyta, the rare distribution area consist of species belonging to the genus Carteria, while the common distribution area consist of species belonging to the genera Monoraphidium, Quadrigula, and Willea.

The fact that Western Mediterranean (43 taxa), Firat-Dicle (41 taxa) and Asi (38 taxa) are the basins with the most new records may be due to the wetlands in 
Table 2. List of phytoplankton taxa identified as new records in 25 basins of Turkey.

Abbreviations; For Basin(s); AK: Akarçay, AN: Antalya, AR: Aras, AS: Asi, WM: Western Mediterranean, EM: Eastern Mediterranean, WB: Western Black Sea, EB: Eastern Black Sea, KM: Küçük Menderes, CE: Ceyhan, CO:

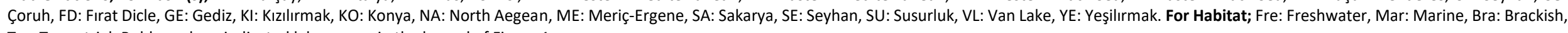
Ter: Terrestrial. Bold numbers indicated lake names in the legend of Figure 1.

\begin{tabular}{|c|c|c|c|c|c|c|}
\hline Taxa & Synonym(s) & $\begin{array}{c}\text { Dimensions (cell) } \\
\text { length } \mathrm{x} \text { weight or } \\
\text { diameter }(\mu \mathrm{m})\end{array}$ & Habitat & $\begin{array}{c}\text { Basin(s) in } \\
\text { Turkey }\end{array}$ & $\begin{array}{c}\text { Lake } \\
\text { Number* }\end{array}$ & \begin{tabular}{|c|}
$\begin{array}{c}\text { Distribution } \\
\text { in the } \\
\text { World }\end{array}$ \\
\end{tabular} \\
\hline \multicolumn{7}{|l|}{ CYANOBACTERIA } \\
\hline Anabaena laxa Braun & - & $4.5-5.0$ & Fre & SA & 217 & Common \\
\hline Anabaena oblonga De Wild. & - & $4.5-5.0$ & Fre & FD & 131 & Rare \\
\hline Anabaena sphaerica var. attenuata Bharadwaja & - & $3.2-5.2$ & Fre & FD & 131 & Rare \\
\hline Anabaenopsis milleri Woron. & - & $3.7-10.9 \times 3.6-8$ & Fre & $\mathrm{YE}$ & 262 & Rare \\
\hline Anabaenopsis rippkae Komárek & - & $4.5-6$ & Fre & FD & 126 & Rare \\
\hline \multirow{2}{*}{ Anathece minutissima (West) Komárek, Kastovsky \& Jezberová } & \multirow{2}{*}{ Microcystis minutissima } & \multirow{2}{*}{$1.5 \times 0.8$} & \multirow{2}{*}{ Fre } & SA & 213 & \multirow{2}{*}{ Common } \\
\hline & & & & FD & 126 & \\
\hline Anathece smithii (Komárk.-Legn. \& Cronberg) Komárek, Kastovsky \& Jezberová & Aphanothece smithii & $3 \times 1.3$ & Fre & SA & 219 & Common \\
\hline \multirow{4}{*}{ Aphanizomenon klebahnii Elenkin } & \multirow{4}{*}{-} & \multirow{4}{*}{$\begin{array}{c}3.9-8.3 \times \\
3.6-4.9\end{array}$} & \multirow{4}{*}{ Fre } & GE & 139,140 & \multirow{4}{*}{ Rare } \\
\hline & & & & NA & $187,189,190$ & \\
\hline & & & & SU & $250,251,254$ & \\
\hline & & & & $\mathrm{CE}$ & 78,91 & \\
\hline Aphanocapsa conferta (West \& G.S.West) Komárk.-Legn. \& Cronberg & Aphanocapsa elachista var. conferta & $1.5-2.0 \mathrm{dia}$ & Fre & FD & 138 & Common \\
\hline Aphanocapsa nubila Komárek \& Kling & Microcystis pulverea var. racemiformis & 1.0-2.0 dia & Fre & FD & 124 & Rare \\
\hline Aphanocapsa planctonica (G.M.Sm.) Komárek \& Anagn. & Microcystis pulverea f. planctonica & 2.0-3.0 dia & Fre & SA & 227 & Rare \\
\hline Aphanothece atrocrustacea Skuja & - & $2-4 \times 1.5-2$ & Fre & WM & 32 & Rare \\
\hline Aphanothece comasii Komárk.-Legn.\& Tavera & - & 1.8-3.0 dia & Fre & WM & 32,33 & Rare \\
\hline Aphanothece floccosa (Zalessky) Cronberg \& Komárek & Microcystis floccosa & $3-4 \times 1-2$ & Fre & WM & 37 & \\
\hline Arthrospira platensis var. non-constricta (Banerji) Desikachary & Spirulina platensis var. non-constricta & $2-3.5$ & Fre & $\mathrm{AK}$ & 2 & Rare \\
\hline Chroococcus mipitanensis (Wolsz.) Geitler & Chroococcus turgidus var. mipitanensis & $1.7-2.5$ & Fre & WM & 32 & Rare \\
\hline Chroococcus lithophilus Erceg. & Gloeocapsa lithophila & 3-5 dia & Fre & WM & 33 & Rare \\
\hline Coelosphaerium aerugineum Lemmerm. & - & $2.2-3.5 \mathrm{dia}$ & Fre, Bra & $\mathrm{FD}$ & 137 & Rare \\
\hline Dolichospermum fallax (Komárek \& Komárk.-Legn.) Wacklin, Hoffm. \& Komárek & Anabaena fallax & $5.2-6.0$ & Fre & FD & 131 & Rare \\
\hline Dolichospermum lemmermannii (Richt.) Wacklin, Hoffm. \& Komárek & Anabaena lemmermannii & $6.3 \times 5$ & Fre & AR & 21 & Common \\
\hline Gloeothece subtilis Skuja & - & $1.5 \times 0.7$ & Fre & AS & 29 & Rare \\
\hline Leptolyngbya ectocarpi (Gomont) Anagn. \& Komárek & Phormidium ectocarpi & $2.0 \times 1.6$ & Fre, Mar & $\mathrm{KO}$ & 170,181 & Rare \\
\hline Limnospira fusiformis (Woron.) Nowicka-Krawczyk, Mühlsteinová \& Haue & Spirulina fusiformis & $4-5$ & Fre & SA & 214 & Rare \\
\hline \multirow{2}{*}{ Merismopedia hyalina (Ehrenb.) Kütz. } & \multirow{2}{*}{ Gonium hyalinum } & \multirow{2}{*}{ 2-3 dia } & \multirow{2}{*}{ Fre, Bra } & WM & 33 & \multirow{2}{*}{ Rare } \\
\hline & & & & SA & 214 & \\
\hline Microcystis botrys Teiling & - & 6-7 dia & Fre & NA & 190 & Common \\
\hline Nodularia sphaerocarpa Bornet \& Flahault & Nodularia harveyana var. sphaerocarpa & $5-7 \times 3.4-6$ & Fre & SA & 222 & Common \\
\hline \multirow{2}{*}{ Nostoc caeruleum Lyngb. } & \multirow[t]{2}{*}{ 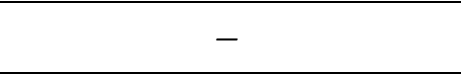 } & \multirow{2}{*}{ 5-7 dia } & \multirow{2}{*}{ Fre } & KO & $169,172,174,176$ & \multirow{2}{*}{ Common } \\
\hline & & & & AN & $11,12,15$ & \\
\hline Oscillatoria chlorina f. perchlorina (Lauterborn) Elenkin & Oscillatoria chlorina var. perchlorina & $4-8 \times 3.5-4$ & Fre & FD & 133 & Rare \\
\hline Oscillatoria trichoides Szafer & - & $5 \times 1-1.5$ & Fre & AK & 5 & Common \\
\hline \multirow{2}{*}{ Pannus spumosus Hickel } & \multirow{2}{*}{-} & \multirow{2}{*}{$1-1.5$ dia } & \multirow{2}{*}{ Fre, Mar } & SA & 226 & Rare \\
\hline & & & & VL & 259 & \\
\hline Phormidium schultzii (Lemmerm.) Anagn. \& Komárek & Oscillatoria schultzii & 3.3 dia & Fre & FD & 127 & Rare \\
\hline
\end{tabular}




\begin{tabular}{|c|c|c|c|c|c|c|}
\hline Taxa & Synonym(s) & $\begin{array}{l}\text { Dimensions (cell) } \\
\text { length } x \text { weight or } \\
\text { diameter }(\mu \mathrm{m})\end{array}$ & Habitat & $\begin{array}{l}\text { Basin(s) in } \\
\text { Turkey }\end{array}$ & $\begin{array}{c}\text { Lake } \\
\text { number* }\end{array}$ & $\begin{array}{l}\begin{array}{c}\text { Distribution } \\
\text { in the } \\
\text { World** }\end{array} \\
\end{array}$ \\
\hline Planktolyngbya microspira Komárek \& Cronberg & - & $1.5-2 \times 1.0$ & Fre & $\mathrm{KM}$ & 195 & Rare \\
\hline \multirow{4}{*}{ Planktothrix isothrix (Skuja) Komárek \& Komárk. } & \multirow{4}{*}{ Oscillatoria agardhii var. isothrix } & \multirow{4}{*}{$1.2-2.5 \times 4-6$} & \multirow{4}{*}{ Fre } & $\mathrm{YE}$ & 271 & \multirow{4}{*}{ Rare } \\
\hline & & & & AK & 2,3 & \\
\hline & & & & & 256 & \\
\hline & & & & $\mathrm{VL}$ & 260 & \\
\hline \multirow{2}{*}{ Pulvinularia suecica Borzì } & \multirow{2}{*}{-} & \multirow{2}{*}{$3.2-6.9 \times 3.0-6.4$} & \multirow{2}{*}{ Fre } & SA & 216 & \multirow{2}{*}{ Rare } \\
\hline & & & & FD & 127 & \\
\hline Romeria gracilis (Koczw.) Koczw. & Raciborskia gracilis & $4.0 \times 1.3$ & Fre & WM & 33 & Rare \\
\hline \multirow{3}{*}{ Rhabdoderma lineare Schmidle \& Lauterborn } & \multirow{3}{*}{ Rhabdoderma lineare var. spirale } & \multirow{3}{*}{$6 \times 2$} & \multirow{3}{*}{ Fre } & KO & 176 & \multirow{3}{*}{ Common } \\
\hline & & & & SA & 211 & \\
\hline & & & & AK & 2 & \\
\hline Snowella fennica Komárek \& Komárk.-Legn. & - & $3.5 \times 2.0$ & Fre & AK & 5 & Rare \\
\hline Snowella litoralis (Häyrén) Komárek \& Hindák & Gomphosphaeria litoralis & $2-3(-4)$ dia & Fre & WM & 33,37 & Rare \\
\hline Synechocystis parvula Perfiliev & - & $0.7-0.9 \mathrm{dia}$ & Fre, Mar & SA & 213 & Rare \\
\hline Woronichinia botrys (Skuja) Komárek \& Hindák & Gomphosphaeria botrys & $5.5 \times 4.5$ & Fre & SA & 224 & Rare \\
\hline \multirow{2}{*}{ Woronichinia karelica Komárek \& Komárk.-Legn. } & \multirow{2}{*}{ (2) } & \multirow{2}{*}{$3.2 \times 1.7$} & \multirow{2}{*}{ Fre } & AK & 5 & \multirow{2}{*}{ Rare } \\
\hline & & & & FD & 124 & \\
\hline \multicolumn{7}{|l|}{ EUGLENOZOA } \\
\hline Anisonema prosgeobium Skuja & - & $30 \times 20$ & Fre & WM & $32,34,35,36,37,42$ & Rare \\
\hline Astasia cylindrica From. & - & $18 \times 10$ & Fre & FD & 138 & Rare \\
\hline Astasia thiophila Hub.-Pest. & - & $22 \times 8$ & Fre & FD & 129 & Rare \\
\hline \multirow{2}{*}{ Euglena antefossa Johnson } & \multirow{2}{*}{-} & \multirow{2}{*}{$175 \times 20$} & Fre & AS & 29 & Rare \\
\hline & & & Pre & WM & 31 & Rare \\
\hline Euglena gracilis var. urophora Chadef. \& Provasoli & - & $70 \times 14$ & Fre & SA & 219 & Rare \\
\hline Euglena mainxii Deflandre & Euglena reticulata & $33 \times 13$ & Fre & AS & 28 & Rare \\
\hline Euglena rostrata Ehrenb. & - & $45 \times 10$ & Fre & SA & 233 & Rare \\
\hline Euglena sacculiformis Schiller & - & $30 \times 14$ & Fre & AK & 1 & Rare \\
\hline & & & & AN & 12 & \\
\hline & & & & $\mathrm{KI}$ & 159,160 & \\
\hline Euglena velata G.A. Klebs & - & $96 \times 25$ & Fre & KO & $169,172,174,175$ & Common \\
\hline & & & & SE & $\begin{array}{c}235,236,237,243, \\
244\end{array}$ & \\
\hline Eualenaria anabaena (Mainx) Karnkowska \& E W Linton & Euglena anabaena & $42 \times 18$ & Fre & AS & 28 & Common \\
\hline 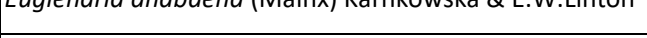 & . & $42 \times 10$ & 然 & FD & 135,138 & C \\
\hline Euglenopsis vacuolata (Skuja) Popova & Astasia vacuolata & $35 \times 13$ & Fre & FD & 138 & Rare \\
\hline Lepocinclis conica (P.Allorge \& Lefèvre) Zakryś \& Lukom. & Lepocinclis ovum var. conica & $18 \times 13$ & Fre & SA & 213 & Rare \\
\hline Lepocinclis fusiformis var. amphirhynchus Nygaard & - & $19 \times 13$ & Fre & SA & 232 & Rare \\
\hline Lepocinclis lobata Conrad & - & $27 \times 25$ & Fre & WM & 36 & Rare \\
\hline Lepocinclis nayalii Conrad & - & $44 \times 24$ & Fre & AS & 28 & Rare \\
\hline Lepocinclis ovum var. angustatum (Deflandre) Conrad & Lepocinclis bütschli var. angustata & $32 \times 17$ & Fre & WM & 32 & Rare \\
\hline Lepocinclis ovum var. dimidio-minor (Deflandre) Conrad & - & $28 \times 15$ & Fre & AS & 29 & Rare \\
\hline Lepocinclis teres f. parvula Conrad & - & $23 \times 16$ & Fre & AK & 1 & Rare \\
\hline Lepocinclis texta var. mammillata (Da Cunha) Conrad & - & $35 \times 21$ & Fre & SA & $213,219,227$ & Rare \\
\hline Lepcrim & - & $30 \times 21$ & rie & WM & 42 & Naie \\
\hline
\end{tabular}




\begin{tabular}{|c|c|c|c|c|c|c|}
\hline Taxa & Synonym(s) & $\begin{array}{l}\text { Dimensions (cell) } \\
\text { length } x \text { weight or } \\
\text { diameter }(\mu \mathrm{m})\end{array}$ & Habitat & $\begin{array}{l}\text { Basin(s) in } \\
\text { Turkey }\end{array}$ & $\begin{array}{c}\text { Lake } \\
\text { number* }\end{array}$ & $\begin{array}{l}\text { Distribution } \\
\text { in the } \\
\text { World** }\end{array}$ \\
\hline Menoidium semilunare var. regulare Wermel & - & $29 \times 11$ & Fre & AS & 29 & Rare \\
\hline Monomorphina aenigmatica (Drezep.) Nudel. \& Triemer & Phacus striatus & $36 \times 9$ & Fre & $\mathrm{KI}$ & 160 & Rare \\
\hline Petalomonas applanata Skuja & - & $27.5 \times 28$ & Fre & FD & 123 & Rare \\
\hline Phacus agilis var. inversus Bourr. & - & $14 \times 10$ & Fre & SA & 233 & Rare \\
\hline \multirow{2}{*}{ Phacus applanatus Pochm. } & \multirow{2}{*}{-} & \multirow{2}{*}{$40 \times 18$} & \multirow{2}{*}{ Fre } & AK & 2 & \multirow{2}{*}{ Rare } \\
\hline & & & & $\mathrm{KO}$ & 174 & \\
\hline Phacus carinatus Skvortsov & - & $35 \times 27$ & Fre & FD & 135 & Common \\
\hline \multirow{9}{*}{ Phacus caudatus Hübner } & \multirow{9}{*}{ Phacus ovalis } & \multirow{9}{*}{$50 \times 27$} & \multirow{9}{*}{ Fre } & AN & 19 & \multirow{9}{*}{ Common } \\
\hline & & & & AS & 23,28 & \\
\hline & & & & NA & 186 & \\
\hline & & & & GE & 141 & \\
\hline & & & & $\mathrm{KO}$ & 170 & \\
\hline & & & & WM & 33 & \\
\hline & & & & WB & 49 & \\
\hline & & & & SU & 247 & \\
\hline & & & & $\mathrm{ME}$ & $206,208,210$ & \\
\hline Phacus circumflexus Pochm. & - & $70 \times 30$ & Fre & WM & 42 & Rare \\
\hline \multirow{2}{*}{ Phacus dangeardii Lemmerm. } & \multirow{2}{*}{-} & \multirow{2}{*}{$16 \times 9$} & \multirow{2}{*}{ Fre } & SA & 225 & \multirow{2}{*}{ Rare } \\
\hline & & & & WM & 32 & \\
\hline Phacus formosus Pochm. & - & $30 \times 17$ & Fre & SA & 223 & Rare \\
\hline Phacus minutus (Playfair) Pochm. & Phacus pleuronectes var. minutus & $28 \times 25$ & Fre & SA & 212 & Rare \\
\hline Phacus swirenkoi Skvortsov & - & $28 \times 19$ & Fre & EM & 106 & Rare \\
\hline Phacus tortuosus Y.V.Roll & - & $29 \times 23$ & Fre & WM & 41 & Rare \\
\hline Strombomonas borystehniensis (Y.V.Roll) T.G.Popova & Trachelomonas borystehniensis & $33 \times 24$ & Fre & SU & 247 & Rare \\
\hline Strombomonas acuminata var. amphora (Playfair) Deflandre & - & $42 \times 21$ & Fre & AS & 28 & Rare \\
\hline \multirow{2}{*}{ Strombomonas acuminata var. deflandreana Conrad } & \multirow{2}{*}{-} & \multirow{2}{*}{$28 \times 15$} & \multirow{2}{*}{ Fre } & FD & 136 & \multirow{2}{*}{ Rare } \\
\hline & & & & SA & 219 & \\
\hline Strombomonas inconstans (N.Carter) Deflandre & - & $20 \times 10$ & Fre & SA & 216 & Rare \\
\hline \multirow{2}{*}{ Strombomonas lanceolata (Playfair) Deflandre } & \multirow{2}{*}{ Trachelomonas lanceolata } & \multirow{2}{*}{$31 \times 20$} & Fre & AS & 28 & Bare \\
\hline & & & rite & FD & 136 & nale \\
\hline Strombomonas napiformis (Playfair) Deflandre & Trachelomonas napiformis & $40 \times 20$ & Fre & WM & 33 & Rare \\
\hline Strombomonas praeliaris var. nana (Palmer) Deflandre & - & $23 \times 13$ & Fre & $\mathrm{FD}$ & 135 & Rare \\
\hline Strombomonas rotunda f. hortobagyi Hub.-Pest. & - & $35 \times 18$ & Fre & AS & 29 & Rare \\
\hline Strombomonas subcurvata var. africana Bourr. \& Gayr. & - & $22 \times 14$ & Fre & WM & 31 & Rare \\
\hline Strombomonas treubii (Wolosz.) Deflandre & Trachelomonas treubii & $22 \times 12$ & Fre & AK & 3 & Rare \\
\hline Strombomonas urceolata (A.Stokes) Deflandre & Trachelomonas urceolata & $41 \times 21$ & Fre & FD & 136 & Rare \\
\hline Strombomonas aspera (Skvortsov) Deflandre & Trachelomonas rhombus & $25 \times 11$ & Fre & SA & 233 & Rare \\
\hline Trachelomonas abrupta f. angustata Deflandre & - & $30 \times 15$ & Fre & FD & 138 & Rare \\
\hline Trachelomonas amphora Svirenko & - & $20 \times 12$ & Fre & KO & 174 & Rare \\
\hline Trachelomonas anulifera Hub.-Pest. & - & $13 \times 12$ & Fre & SA & 219 & Rare \\
\hline Trachelomonas bacillifera f. sparsispina Deflandre & - & $26 \times 22$ & Fre & FD & 133 & Rare \\
\hline Trachelomongs chodati Skvortsov & - & $18 \times 10$ & Fre & FD & 134 & Rare \\
\hline 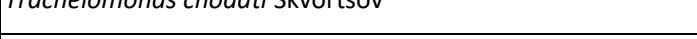 & - & $10 \times 10$ & rite & SA & 227 & nare \\
\hline Trachelomonas columba Palmer & - & $27 \times 26$ & Fre & $\mathrm{KO}$ & 170,173 & Rare \\
\hline
\end{tabular}




\begin{tabular}{|c|c|c|c|c|c|c|}
\hline Taxa & Synonym(s) & $\begin{array}{l}\text { Dimensions (cell) } \\
\text { length } \mathrm{x} \text { weight or } \\
\text { diameter }(\mu \mathrm{m}) \\
\end{array}$ & Habitat & $\begin{array}{c}\text { Basin(s) in } \\
\text { Turkey }\end{array}$ & $\begin{array}{c}\text { Lake } \\
\text { number* }\end{array}$ & \begin{tabular}{|c}
$\begin{array}{c}\text { Distribution } \\
\text { in the } \\
\text { World** }\end{array}$ \\
\end{tabular} \\
\hline Trachelomonas curta var. minima Tell \& Zalocar & - & $15 \times 15$ & Fre & AS & 23 & Rare \\
\hline Trachelomonas dangeardii var. glabra (Playfair) Deflandre & Trachelomonas armata var. glabra & $27 \times 20$ & Fre & WM & 32 & Rare \\
\hline Trachelomonas globularis var. boyeri (Palmer) Conrad & - & $22 \times 21$ & Fre & SA & 227 & Rare \\
\hline Trachelomonas grandis Kam.P.Singh & - & $18 \times 16$ & Fre & FD & 123 & Rare \\
\hline Trachelomonas granulosa var. subglobosa Playfair & - & $27 \times 23$ & Fre & SA & 231 & Rare \\
\hline Trachelomonas hexangulata var. hexagona (Oye) Hub.-Pest. & Trachelomonas hexagona & $26 \times 23$ & Fre & AS & 27 & Rare \\
\hline Trachelomonas heduma Conrad & - & $21 \times 18$ & Fre & SA & 233 & Rare \\
\hline Trachelomonas horrida Palmer & - & $30 \times 24$ & Fre & SE & 236 & Rare \\
\hline Trachelomonas kelloggii var. nana Balech & - & $53 \times 50$ & Fre & WM & 41 & Rare \\
\hline Trachelomonas komarowii Skvortsov & - & $18 \times 17$ & Fre & FD & 123 & Rare \\
\hline Trachelomonas lismorensis var. inermis Playfair & - & $20 \times 16$ & Fre & FD & 137 & Rare \\
\hline Trachelomonas perlata Deflandre & - & $13 \times 11$ & Fre & SA & 219 & Rare \\
\hline Trachelomonas pseudofelix Deflandre & - & $13 \times 12$ & Fre & SA & 225,232 & Rare \\
\hline Trachelomonas oblonga var. australica Playfair & Trachelomonas planctonica var. australica & $11 \times 10$ & Fre & SA & 232 & Rare \\
\hline \multirow{4}{*}{ Trachelomonas obovata var. klebsiana Deflandre } & \multirow{4}{*}{ Trachelomonas obovata f. klebsiana } & \multirow{4}{*}{$26 \times 18$} & \multirow{4}{*}{ Fre } & AK & 10 & \multirow{4}{*}{ Rare } \\
\hline & & & & AS & 24 & \\
\hline & & & & FD & 124,133 & \\
\hline & & & & SA & 219 & \\
\hline Trachelomonas orenburgika var. ornata Skvortsov & - & $17 \times 14$ & Fre & FD & 129 & Rare \\
\hline Trachelomonas stokesiana f. meandrina (Conrad) T.G.Popova & Trachelomonas rugulosa f. meandrina & $18 \times 17$ & Fre & SA & 233 & Rare \\
\hline Trachelomonas rugulosa var. obliqua Bourr. & - & $18 \times 17$ & Fre & FD & 134 & Rare \\
\hline Trachelomonas scabra var. coberensis Deflandre & - & $14 \times 13$ & Fre & SA & 227 & Rare \\
\hline Trachelomonas scabra var. ovata f. minör Playfair & - & $22 \times 16$ & Fre & FD & 138 & Rare \\
\hline Trachelomonas sydneyensis var. minima Playfair & - & $26 \times 19$ & Fre & SA & 216 & Rare \\
\hline Trachelomonas sydneyensis var. obesa Playfair & - & $28 \times 22$ & Fre & SA & 224 & Rare \\
\hline Trachelomonas tuberculata Middelh. & - & $10 \times 10$ & Fre & AS & 23 & Rare \\
\hline Trachelomonas varians f. globosa Deflandre & - & $16 \times 16$ & Fre & FD & 130 & Rare \\
\hline \multirow{3}{*}{ Trachelomonas verrucosa f. irregularis Deflandre } & \multirow{3}{*}{-} & \multirow{3}{*}{$14 \times 14$} & \multirow{3}{*}{ Fre } & AS & 26 & \multirow{3}{*}{ Rare } \\
\hline & & & & FD & 138 & \\
\hline & & & & SA & 219 & \\
\hline \multirow{2}{*}{ Trachelomonas verrucosa f. sparseornata Deflandre } & \multirow{2}{*}{-} & \multirow{2}{*}{$19 \times 19$} & \multirow{2}{*}{ Fre } & AK & 3 & \multirow{2}{*}{ Rare } \\
\hline & & & & FD & 129 & \\
\hline Trachelomonas verrucosa var. macrotuberculata Grand. & - & $24 \times 23$ & Fre & SA & 227 & Rare \\
\hline Trachelomonas verrucosa var. spirogyra (Bal.) Hub.-Pest. & Trachelomonas spirogyra & $13 \times 12$ & Fre & SA & 219,227 & Rare \\
\hline Trachelomonas volvocina var. derephora Conrad & Trachelomonas derephora & $16 \times 15$ & Fre & FD & 137 & Rare \\
\hline Trachelomonas volvocinopsis var. khannae (Skvor.) Bourr. & - & $29 \times 29$ & Fre & AS & 27 & Rare \\
\hline Trachelomonas zorensis Deflandre & - & $22 \times 16$ & Fre & AS & 29 & Rare \\
\hline \multicolumn{7}{|l|}{ CHLOROPHYTA } \\
\hline Acanthosphaera zachariasii Lemmerm. & Acanthosphaera tenuispina & 8-15 dia & Fre & AS & 24 & Common \\
\hline Ankistrodesmus stipitatus Komárk.-Legn. & Ankistrodesmus falcatus var. stipitatus & $30-45 \times 3-6$ & Fre & FD & 129 & Common \\
\hline Aulacomonas submarina Skuja & - & $6 \times 2$ & Fre, Mar & SA & 229,231 & Common \\
\hline Carteria agloeformis Nygaard & - & $10-45$ dia & Fre & FD & 138 & Rare \\
\hline Carteria fritschii H.Takeda & - & 6-16 dia & Fre & FD & 133,138 & Rare \\
\hline Carteria huberi Christen & - & $18-26 \times 10-16$ & Fre, Mar & SA & 227 & Rare \\
\hline
\end{tabular}




\begin{tabular}{|c|c|c|c|c|c|c|}
\hline Taxa & Synonym(s) & $\begin{array}{l}\text { Dimensions (cell) } \\
\text { length } x \text { weight or } \\
\text { diameter }(\mu \mathrm{m}) \\
\end{array}$ & Habitat & $\begin{array}{l}\text { Basin(s) in } \\
\text { Turkey }\end{array}$ & $\begin{array}{c}\text { Lake } \\
\text { number* }\end{array}$ & \begin{tabular}{|c}
$\begin{array}{c}\text { Distribution } \\
\text { in the } \\
\text { World** }\end{array}$ \\
\end{tabular} \\
\hline Carteria lohammari Skuja & - & $14-18 \times 8-10$ & Fre & FD & 138 & Rare \\
\hline \multirow{2}{*}{ Carteria inversa (Korshikov) Bourr. } & \multirow{2}{*}{ Carteria crucifera var. inversa } & \multirow{2}{*}{$22-30 \times 16-24$} & \multirow{2}{*}{ Fre } & FD & 127,138 & \multirow{2}{*}{ Rare } \\
\hline & & & & AS & 27 & \\
\hline Carteria sphaerica Hub.-Pest. & - & $13-14 \times 9$ & Fre & SA & 216 & Rare \\
\hline Carteria stellifera Nygaard & - & $8-14 \times 10-12$ & Fre & FD & 130 & Rare \\
\hline Characium angustum Braun & Characium apiculatum & $70-170 \times 2.5-6$ & Fre & AK & 4 & Common \\
\hline Chlamydomonas anglica (G.S. West) Pascher & - & $14-20 \times 9-13$ & Fre & SA & 222 & Common \\
\hline \multirow{2}{*}{ Chlamydomonas bichlora Pascher \& Jahoda } & \multirow{2}{*}{-} & \multirow{2}{*}{$13-18 \times 5-7$} & \multirow{2}{*}{ Fre } & AS & 26 & \multirow{2}{*}{ Rare } \\
\hline & & & & SA & 224 & \\
\hline Chlamydomonas confinis Skuja & - & $13-19 \times 9-14$ & Fre & FD & 137 & Rare \\
\hline Chlamydomonas conocylindrus Pascher & - & $12-17 \times 9-12$ & Fre & SA & 222 & Rare \\
\hline Chlamydomonas crassa Christen & Chloromonas crassa & $10-17 \times 11-16$ & Fre & AN & 19 & Rare \\
\hline Chlamydomonas elegans G.S. West & - & $12-14 \times 7-10$ & Fre & SA & 224 & Rare \\
\hline Chlamydomonas gloeophila Skuja & - & $14-17 \times 4-6$ & Fre & $\mathrm{AK}$ & 2 & Rare \\
\hline Chlamydomonas granulosa Skvortsov & - & $22 \times 12$ & Fre & WM & 42 & Rare \\
\hline \multirow{3}{*}{ Chlamydomonas incerta Pascher } & \multirow{3}{*}{$\begin{array}{c}\text { Chlamydomonas incerta var. } \\
\text { macropyrenoidosa }\end{array}$} & \multirow{3}{*}{$27 \mathrm{dia}$} & \multirow{3}{*}{ Fre } & SA & $\begin{array}{c}213,215,218,219, \\
225,233\end{array}$ & \multirow{3}{*}{ Rare } \\
\hline & & & & AK & $1,2,3,4,5,10$ & \\
\hline & & & & FD & 135,138 & \\
\hline \multirow{3}{*}{ Chlamydomonas incisa Korshikov } & \multirow{3}{*}{-} & \multirow{3}{*}{$24-34 \times 10-22$} & \multirow{3}{*}{ Fre } & SA & 231 & \multirow{3}{*}{ Rare } \\
\hline & & & & AK & 3 & \\
\hline & & & & FD & 136 & \\
\hline Chlamydomonas klinobasis Skuja & Chlamydomonadopsis klinorostris & $13-17 \times 10-17$ & Fre & AS & 24 & Rare \\
\hline Chlamydomonas lapponica Skuja & - & $8-10 \times 22$ & Fre & KO & 175 & Rare \\
\hline \multirow{2}{*}{ Chlamydomonas macroplastida Lund } & \multirow{2}{*}{-} & \multirow{2}{*}{$15-25 \times 8-17$} & Fre Ter & SA & 215 & Rare \\
\hline & & & He, rei & AK & 2 & \\
\hline Chlamydomonas macropyrenoidosa Skuja & - & $12-15 \times 8-10$ & Fre & WM & 33 & Rare \\
\hline Chlamydomonas metapyrenigera Skuja & - & $15-23 \times 5-10$ & Fre & $\mathrm{FD}$ & 124,130 & Rare \\
\hline & & & & SA & 220,229 & \\
\hline Chlamydomonas microsphaerella Pascher \& Jahoda & - & $12-14 \times 6-8$ & Fre & AK & 2 & Rare \\
\hline & & & & FD & 130 & \\
\hline Chlamvdomonas nivalis (F. A Bauer) Wille & Protococrus nivalis & $12 \times 6$ & Fre Ter & SA & 215 & Common \\
\hline 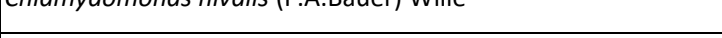 & 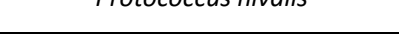 & $12 \times 0$ & Hie, rei & AS & 24 & Comint \\
\hline Chlamydomonas penium Pascher & - & $10-15 \times 5-10$ & Fre & AS & 26 & Rare \\
\hline Chlamydomonas pulvinata Vischer & - & $12-16 \times 6-8$ & Fre & $\mathrm{AK}$ & 5 & Rare \\
\hline Chlamydomonas rhopaloides Korshikov & - & $11-13 \times 5-8$ & Fre & SA & 229 & Rare \\
\hline Chlamydomonas rotifera Gerloff & - & $12-16 \times 10-12$ & Fre & WM & 39 & Rare \\
\hline Chlamydomonas simplex Pascher & - & $11-14 \times 7-10$ & Fre & FD & 134 & Rare \\
\hline Chlamydomonas skujae Pascher & - & $5.5-7 \times 6-8$ & Fre & AS & 28 & Rare \\
\hline Chlamydomonas tremulans Skuja & - & $6.5-11.5 \times 4-8.5$ & Fre & WM & 32 & Rare \\
\hline Chlamydomonas truncata Pascher \& Jahoda & - & $10-14 \times 6-9$ & Fre & WM & 38 & Rare \\
\hline Chlamydomonas upsaliensis Skuja & - & $10-20 \times 11-17$ & Fre & SA & 215,225 & Rare \\
\hline Chiamyaomonas upsaliensis skuja & - & $10-20 \times 11-17$ & (I) & WM & 32,42 & Rare \\
\hline Chlorella chlorelloides (Naumann) C.Bock, Krienitz \& Pröschold & Dictyosphaerium chlorelloides & $3-7 \times 2-6$ & Fre & SU & 246 & Common \\
\hline & & & & ME & 210 & \\
\hline
\end{tabular}


Table 2. Continued

\begin{tabular}{|c|c|c|c|c|c|c|}
\hline Taxa & Synonym(s) & $\begin{array}{c}\text { Dimensions (cell) } \\
\text { length } x \text { weight or } \\
\text { diameter }(\mu \mathrm{m})\end{array}$ & Habitat & $\begin{array}{c}\text { Basin(s) in } \\
\text { Turkey }\end{array}$ & $\begin{array}{c}\text { Lake } \\
\text { number* }\end{array}$ & \begin{tabular}{|c|c}
$\begin{array}{c}\text { Distribution } \\
\text { in the } \\
\text { World** }\end{array}$ \\
\end{tabular} \\
\hline Chlorolobion lunulatum Hindák & Keratococcus lunulatus & $10 \times 2-4$ & Fre, Ter & AS & 29,30 & Common \\
\hline \multirow{3}{*}{ Chloromonas vernalis (Skuja) Nakada } & \multirow{3}{*}{ Chloromonas tapeta var. vernalis } & \multirow{3}{*}{$15-25 \times 11-24$} & \multirow{3}{*}{ Fre } & SA & 223 & \multirow{3}{*}{ Rare } \\
\hline & & & & AK & 5 & \\
\hline & & & & FD & 135 & \\
\hline Chloromonas vesterbottnica (Skuja) Gerloff \& H.Ettl & Chlamydomonas vesterbottnica & $20-23 \times 11-18$ & Fre & WM & 32 & Rare \\
\hline Chloromonas westiana (Pascher) Gerloff \& Ettl & Chlamydomonas westiana & $12-14 \times 10-12$ & Fre & AS & 26 & Rare \\
\hline Coccomonas platyformis Jane & - & $12-20 \times 16-20$ & Fre & SA & 229 & Rare \\
\hline \multirow{2}{*}{ Coenococcus planctonicus Korshikov } & \multirow{2}{*}{ Eutetramorus planctonicus } & \multirow{2}{*}{$5.5-6 \mathrm{dia}$} & \multirow{2}{*}{ Fre } & FD & 134 & \multirow{2}{*}{ Rare } \\
\hline & & & & AS & 28 & \\
\hline \multirow{2}{*}{ Desmatractum indutum (Geitler) Pascher } & \multirow{2}{*}{ Calyptobactron indutum } & \multirow{2}{*}{$3-5 \times 7-10$} & \multirow{2}{*}{ Fre } & AK & 6 & \multirow{2}{*}{ Rare } \\
\hline & & & & AS & 23 & \\
\hline Desmodesmus abundans var. brevicauda (G.M. Sm.) Taskin \& Alp & Scenedesmus subspicatus var. brevicauda & $5-7 \times 2-3$ & Fre & EM & $\begin{array}{c}103,104,105,106, \\
110\end{array}$ & Common \\
\hline \multirow{2}{*}{ Dicellula geminata (Printz) Korshikov } & \multirow{2}{*}{ Dicellula planctonica } & \multirow{2}{*}{$10-12 \times 6-7$} & \multirow{2}{*}{ Fre } & WM & $31,32,35,41,42$ & \multirow{2}{*}{ Common } \\
\hline & & & & AK & 3 & \\
\hline \multirow{3}{*}{ Dictyosphaerium subsolitarium Goor } & \multirow{3}{*}{-} & \multirow{3}{*}{$1.5-3$ dia } & \multirow{3}{*}{ Fre, Mar } & SA & $225,227,228,232$ & \\
\hline & & & & AK & 10 & Common \\
\hline & & & & FD & 138 & \\
\hline Didvmocystis inermis (Fott) Fott & Scenedesmus inermis & $8-13 \times 3-6$ & Fre & SA & 232 & Common \\
\hline 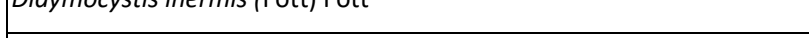 & 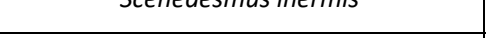 & (2) & (I) & AK & 10 & (U) \\
\hline Didymogenes palatina Schmidle & - & $6-11 \times 1.3-3.7$ & Fre & AS & 28 & Common \\
\hline Eutetramorus tetrasporus Komárek & Coenococcus tetrasporus & 2-3.8 dia & Fre & FD & 127,130 & Rare \\
\hline Franceia javanica (C.Bernard) Hortob. & Chodatella javanica & $4-8 \times 3.5-5$ & Fre & SA & 218 & Rare \\
\hline Gloeomonas tecta (Skuja) H.Ettl \& O.Ettl & Chlamydomonas tecta & $20-32 \times 13-27$ & Fre & SA & 219 & Rare \\
\hline Golenkinia brevispina Korshikov & - & $11-17$ dia & Fre & SA & 220 & Rare \\
\hline Golenkinia maxima Tiffany \& Ahlstrom & - & $17-22$ dia & Fre & SA & 213 & Rare \\
\hline . & & $17-2<$ uid & rite & AS & 28 & naile \\
\hline & & & & WM & 32 & \\
\hline Golenkinia viridis (Frenzel) Printz & Phythelios viridis & 10-12 dia & Fre & SA & 214,219 & Rare \\
\hline & & & & AK & $2,3,9$ & \\
\hline Hafniomonas montana (Geitler) Ettl \& Moestrup & Pyramimonas montana & $12-15 \times 10-12$ & Fre & SA & $224,225,226$ & Rare \\
\hline Hafniomonas reticulata (Korshikov) Ettl \& Moestrup & Pyramimonas reticulata & $10-12 \times 6-9$ & Fre & SA & 227 & Rare \\
\hline Hyalogonium elongatum Matv. & - & $4-10 \times 20-80$ & Fre & AK & 2 & Rare \\
\hline Ixipapillifera sacculiformis (Korshikov) Nakada & Chlamydomonas sacculiformis & $17-24 \times 8-14$ & Fre, Mar & SA & 224 & Rare \\
\hline & & & & WB & 47,50 & \\
\hline Kirchneriella major C.Bernard & Pseudokirchneriella major & $12-20 \times 2.5-6$ & Fre & EM & $\begin{array}{c}103,104,110,111, \\
112 \\
\end{array}$ & Common \\
\hline Micractinium elongatum (H.J. Carter) Hegew. \& Schnepf & Conococcus elongatus & 6-7 dia & Fre & SA & 229 & Rare \\
\hline & corrocuecus etoriguicus & 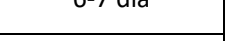 & Fre & AK & 9 & Rare \\
\hline Microglena coccifera (Gorosch.) Demchenko, Mikhailyuk \& Proschold & $\begin{array}{c}\text { Chlamydomonas coccifera var. } \\
\text { mesopyrenigera }\end{array}$ & $22-24 \times 19-23$ & Fre & AS & 27 & Rare \\
\hline Microspora amoena var. gracilis (Wille) De Toni & Microspora elegans & 6-8 dia & Fre & КO & 172 & Rare \\
\hline Monoraphidium obtusum (Korshikov) Komárk.-Legn. & Choricystis obtusa & $32-64 \times 4.5-5$ & Fre & SA & 223 & Common \\
\hline Monoraphidium pseudobraunii (Belcher \& Swale) Heynig & Ankistrodesmus pseudobraunii & $10-25 \times 1.5-2.5$ & Fre & SA & 219 & Common \\
\hline 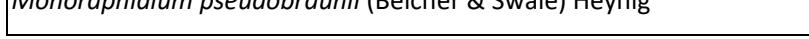 & 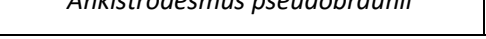 & $10-23 \times 1.5-2.3$ & rite & FD & 129 & 然 \\
\hline
\end{tabular}




\begin{tabular}{|c|c|c|c|c|c|c|}
\hline Taxa & Synonym(s) & $\begin{array}{c}\text { Dimensions (cell) } \\
\text { length } x \text { weight or } \\
\text { diameter }(\mu \mathrm{m})\end{array}$ & Habitat & $\begin{array}{l}\text { Basin(s) in } \\
\text { Turkey }\end{array}$ & $\begin{array}{c}\text { Lake } \\
\text { number* }\end{array}$ & $\begin{array}{l}\text { Distribution } \\
\text { in the } \\
\text { World** }\end{array}$ \\
\hline Mucidosphaerium sphagnale (Hindak) C.Bock, Proschold \& Krienitz & Dictyosphaerium sphagnale & $20 \mathrm{dia}$ & Fre & FD & 131 & Rare \\
\hline Oocystis tainoensis Komárek & - & $3.5-9.5 \times 2-4.8$ & Fre & KO & 184 & Rare \\
\hline \multirow{2}{*}{ Palmococcus hercynicus (H. Heynig) I. Kostikov, T. Darienko, A. Lukesová, \& L. Hoffm. } & \multirow{2}{*}{ Coenocystis hercynica } & \multirow{2}{*}{$10-11 \times 8-9$} & \multirow{2}{*}{ Fre, Ter } & FD & 137 & \multirow{2}{*}{ Rare } \\
\hline & & & & SA & 216 & \\
\hline Palmococcus reniformis (Korshikov) I.Kostikov, T.Darienko, A.Lukesová \& L.Hoffm. & Coenocystis reniformis & $10-12.8 \times 6-7$ & Fre & SA & 227 & Common \\
\hline Papenfussiomonas cordata (Pascher \& Jahoda) Desikachary & Dunaliella cordata & $8-10 \times 12-14$ & Fre & WM & 31 & Rare \\
\hline Paulschulzia pseudovolvox (P.Schultz) Skuja & Schulziella pseudovolvox & 40-350 dia & Fre & SA & 224 & Common \\
\hline Paulschulzia tenera (Korshikov) J.W.G.Lund & Tetraspora tenera & 6-13 dia & Fre & $E B$ & 119 & Rare \\
\hline Pediastrum orbitale Komárek & - & $7-9 \times 9.5-11$ & Fre & FD & 129 & Rare \\
\hline Pediastrum ovatum (Ehrenb.) Braun & Pediastrum simplex var. ovatum & $16-57 \times 6-38$ & Fre & SA & 227 & Common \\
\hline Pediastrum simplex var. clathratum Schröt. & - & $16-57 \times 6-38$ & Fre & AN & 11 & Common \\
\hline Phacotus glaber Pochm. & - & $40-71$ dia & Fre & SA & 231 & Rare \\
\hline Phyllariochloris caeca (Pascher) Pascher \& Jahoda & Phyllariomonas caeca & $12-14 \times 18-26$ & Fre & FD & 122 & Rare \\
\hline Planktococcomyxa lacustris (Chodat) Kostikov, Darienko, Lukesová \& Hoffm. & Coccomyxa lacustris & $6.7-8.3 \times 2.4-3.5$ & Fre & FD & 127 & Common \\
\hline Platymonas cordiformis Korshikov & - & $16-23 \times 14-20$ & Fre & WM & 32,41 & Rare \\
\hline Podohedriella falcata (Düringer) Hindák & Podohedra falcata & $30-68 \times 1.5-2.5$ & Fre & KO & 174 & Rare \\
\hline \multirow{3}{*}{ Pseudodidymocystis fina (Komárek) Hegew. \& Deason } & \multirow{3}{*}{ Choricystis fina } & \multirow{3}{*}{$4.3-7 \times 1.6-2.8$} & \multirow{3}{*}{ Fre } & SA & 214 & \multirow{3}{*}{ Rare } \\
\hline & & & & FD & $\begin{array}{c}126,129,135,136 \\
138\end{array}$ & \\
\hline & & & & AS & $24,28,29$ & \\
\hline Pseudopediastrum subgranulatum (Racib.) Lenarczyk & Pediastrum subgranulatum & $5.5-28.5 \times 5-25$ & Fre & $\mathrm{KI}$ & 151 & Common \\
\hline Pteromonas rugosa Skvortsov & - & $12-25 \times 10-13$ & Fre & WM & 42 & Rare \\
\hline Pyramimonas delicatula B.M.Griffiths & - & $12-15$ dia & Fre, Mar & WM & 32 & Rare \\
\hline Pyramimonas inconstans Hodgetts & - & $7.5-10 \times 10-15$ & Fre, Mar & SA & 216,227 & Rare \\
\hline Pyramimonas splendidissima Pascher & - & $5-7 \times 10-12$ & Fre & SA & 216 & Rare \\
\hline Quadrigula chodatii (Tanner-Füll.) G.M.Sm. & Gregiochloris chodatii & $30-80 \times 3.5-7$ & Fre & SA & 227 & Common \\
\hline Quadrigula sabulosa Hindák & - & $21 \times 1.7-2.3$ & Fre & FD & 130 & Common \\
\hline Radiococcus bavaricus (Skuja) Komárek & Coenochloris bavarica & $6-15$ dia & Fre & FD & 129 & Rare \\
\hline Rayssiella hemisphaerica Edelst. \& Prescott & - & $15-20 \times 6-10$ & Fre & WM & 32 & Rare \\
\hline Scenedesmus aldavei E.H.Hegewald & - & $4.4-8.6 \times 1.9-4.8$ & Fre & SA & 212,216 & Rare \\
\hline \multirow{4}{*}{ Scenedesmus calyptratus Comas } & \multirow{4}{*}{-} & \multirow{4}{*}{$7.4-9.6 \times 2-4$} & \multirow{4}{*}{ Fre } & WM & 32,42 & \multirow{4}{*}{ Rare } \\
\hline & & & & SA & $\begin{array}{c}211,214,215,224, \\
227,232\end{array}$ & \\
\hline & & & & FD & $130,132,133,136$ & \\
\hline & & & & AS & 26 & \\
\hline Scenedesmus formidolosus Hortob. & - & $13.5-16.8 \times 4.2$ & Fre & FD & 138 & Rare \\
\hline Scenedesmus fusiformis Menegh. & - & $13-16 \times 3.6-4.5$ & Fre & WM & 32,42 & Rare \\
\hline Scenedesmus gutwinskii var. bacsensis Uherk. & - & $5.2-13 \times 2-4.5$ & Fre & WM & 32,33 & Rare \\
\hline Scenedesmus opoliensis var. bicaudatus Hortob. & - & $10-21 \times 2.7-6.5$ & Fre & AK & 2,3 & Rare \\
\hline Scenedesmus protuberans f. danubianus Hortob. & - & $11.2-34 \times 3.5-7$ & Fre & AS & 28 & Rare \\
\hline Scenedesmus protuberans var. minor Ley & - & $15-30 \times 5-9$ & Fre & AS & 28 & Rare \\
\hline Scenedesmus pseudoquadricauda Hortob. & - & $15-16.5 \times 5.4-6.5$ & Fre & WM & 32 & Rare \\
\hline Scenedesmus similagineus Hortob. & - & $7.5-20 \times 4-8$ & Fre & WM & 32,41 & Rare \\
\hline Scenedesmus tibiscensis Uherk. & - & $7.5-13 \times 3.5-4.5$ & Fre & $\mathrm{KI}$ & 151,154 & Rare \\
\hline
\end{tabular}




\begin{tabular}{|c|c|c|c|c|c|c|}
\hline Taxa & Synonym(s) & $\begin{array}{l}\text { Dimensions (cell) } \\
\text { length } x \text { weight or } \\
\text { diameter }(\mu \mathrm{m})\end{array}$ & Habitat & $\begin{array}{l}\text { Basin(s) in } \\
\text { Turkey }\end{array}$ & $\begin{array}{c}\text { Lake } \\
\text { number* }\end{array}$ & $\begin{array}{l}\text { Distribution } \\
\text { in the } \\
\text { World** }\end{array}$ \\
\hline Schroederia ecsediensis Hortob. & - & $15-20 \times 1.5-4$ & Fre & FD & 130 & Rare \\
\hline \multirow{2}{*}{ Siderocystopsis punctifera (Boloch.) Hegew. \& Schnepf } & \multirow{2}{*}{ Siderocystopsis fusca } & \multirow{2}{*}{$6-20 \times 4-18$} & \multirow{2}{*}{ Fre } & KO & 175 & \multirow{2}{*}{ Rare } \\
\hline & & & & AS & 28 & \\
\hline Sorastrum spinulosum Nägeli & Sorastrum cornutum & $8-20 \times 6-18$ & Fre & AN & 17 & Rare \\
\hline Sphaerellopsis agloë Pascher & - & $5.5-7 \times 9-11$ & Fre & SA & 227 & Rare \\
\hline Tetraëdron minimum f. apiculatum (Reinsch) De Toni & Polyedrium minimum f. apiculata & $6-20 \times 5-8$ & Fre & AS & 23 & Rare \\
\hline Tetraëdron octaedricum (Reinsch) Hansg. & Polyedrium octaedricum & $32-47$ dia & Fre & FD & 136 & Rare \\
\hline Tetraëdron octaedricum var. spinosum (Reinsch) West \& G.S.West & Polyedrium octaedricum f. spinosum & $38 \times 47$ & Fre & WM & 32 & Rare \\
\hline Tetraëdron trigonum f. minus-obtusum (Reinsch) De Toni & - & $14-16$ & Fre & AS & 28 & Rare \\
\hline Tetraëdron trigonum var. longispinum Philipose & - & $8-18 \times 3-8$ & Fre & SA & 227 & Rare \\
\hline Trochiscia granulata (Reinsch) Hansg. & Acanthococcus granulatus & $8-23$ dia & Fre & WB & 49,55 & Rare \\
\hline \multirow{2}{*}{ Tetraselmis arnoldii (Proshk.-Lavr.) R.E.Norris, Hori \& Chihara } & \multirow{2}{*}{ Platymonas arnoldii } & \multirow{2}{*}{$12-15 \times 9.6-12.5$} & \multirow{2}{*}{ Fre } & $\mathrm{KO}$ & $174,181,184$ & \multirow{2}{*}{ Rare } \\
\hline & & & & AN & 12 & \\
\hline \multirow{3}{*}{ Tetraselmis elliptica (G.M. Sm.) R.E.Norris, Hori \& Chihara } & \multirow{3}{*}{ Platymonas elliptica } & \multirow{3}{*}{$7-8 \times 4-5$} & \multirow{3}{*}{ Fre } & WM & 36,42 & \multirow{3}{*}{ Rare } \\
\hline & & & & SA & $221,222,227$ & \\
\hline & & & & AK & 5 & \\
\hline \begin{tabular}{|l|} 
Volvox rousseletii G.S.West \\
\end{tabular} & - & $16 \times 8$ & Fre & SA & 219 & Rare \\
\hline \multirow{6}{*}{ Willea crucifera (Wolle) D.M.John, M.J.Wynne \& P.M.Tsarenko } & \multirow{6}{*}{ Crucigeniella crucifera } & \multirow{6}{*}{$5-9 \times 3-5$} & \multirow{6}{*}{ Fre } & $\mathrm{KI}$ & 148 & \multirow{6}{*}{ Common } \\
\hline & & & & WM & 32 & \\
\hline & & & & SA & 219 & \\
\hline & & & & $\mathrm{CO}$ & 98 & \\
\hline & & & & FD & $130,131,135,138$ & \\
\hline & & & & AS & 28,29 & \\
\hline
\end{tabular}

Note:*Marașlıoğlu et al. (2021)

**Distribution has been evaluated according to AlgaeBase (Guiry and Guiry, 2021) 

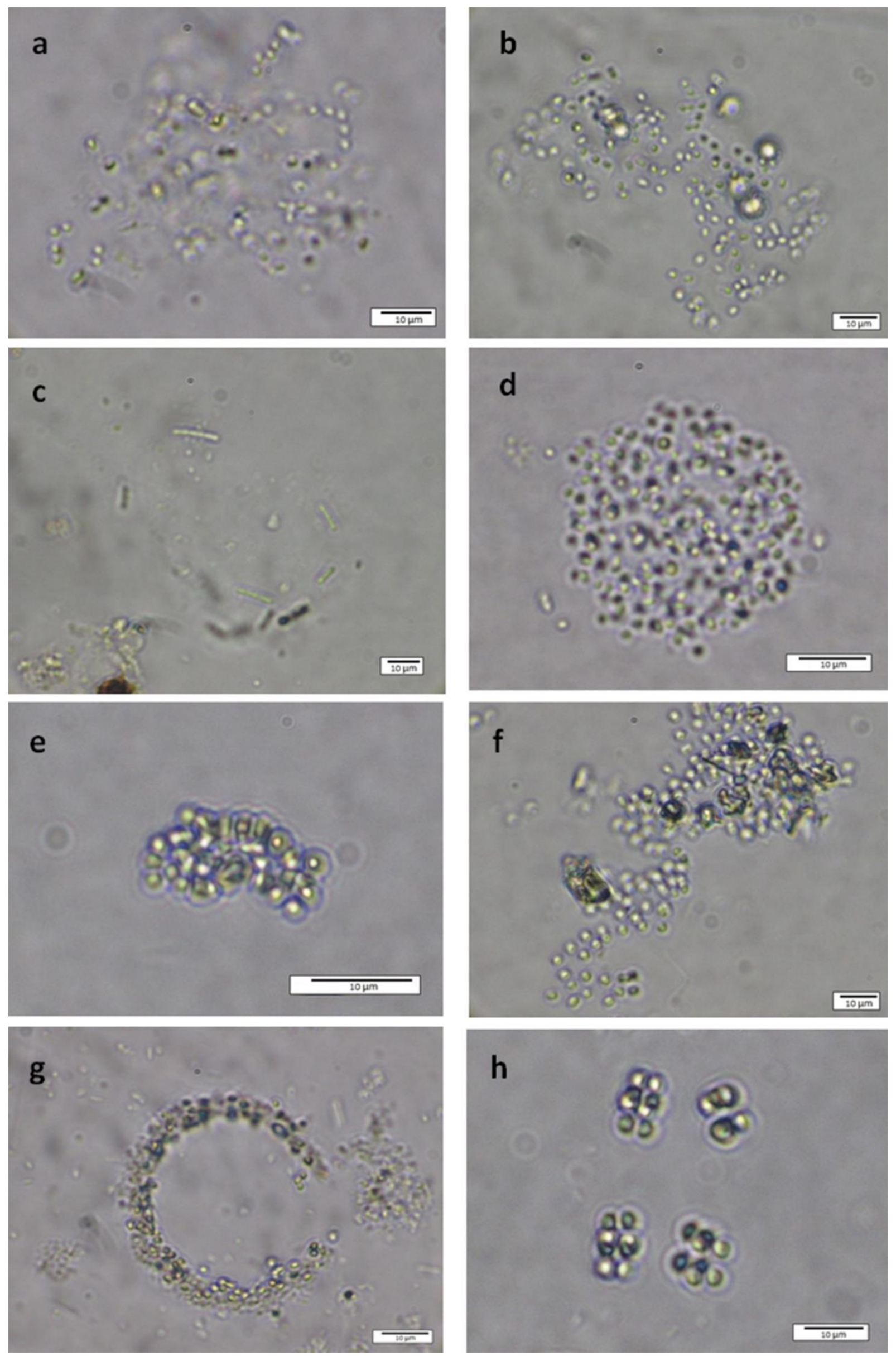

Figure 2. Cyanobacteria; a) Anathece minutissima, b) Anathece smithii, c) Rhabdoderma lineare, d) Aphanocapsa conferta, e) Aphanocapsa nubila, f) Aphanocapsa planctonica, g) Pannus spumosus, h) Merismopedia hyalina, (Scale $10 \mu \mathrm{m})$. 

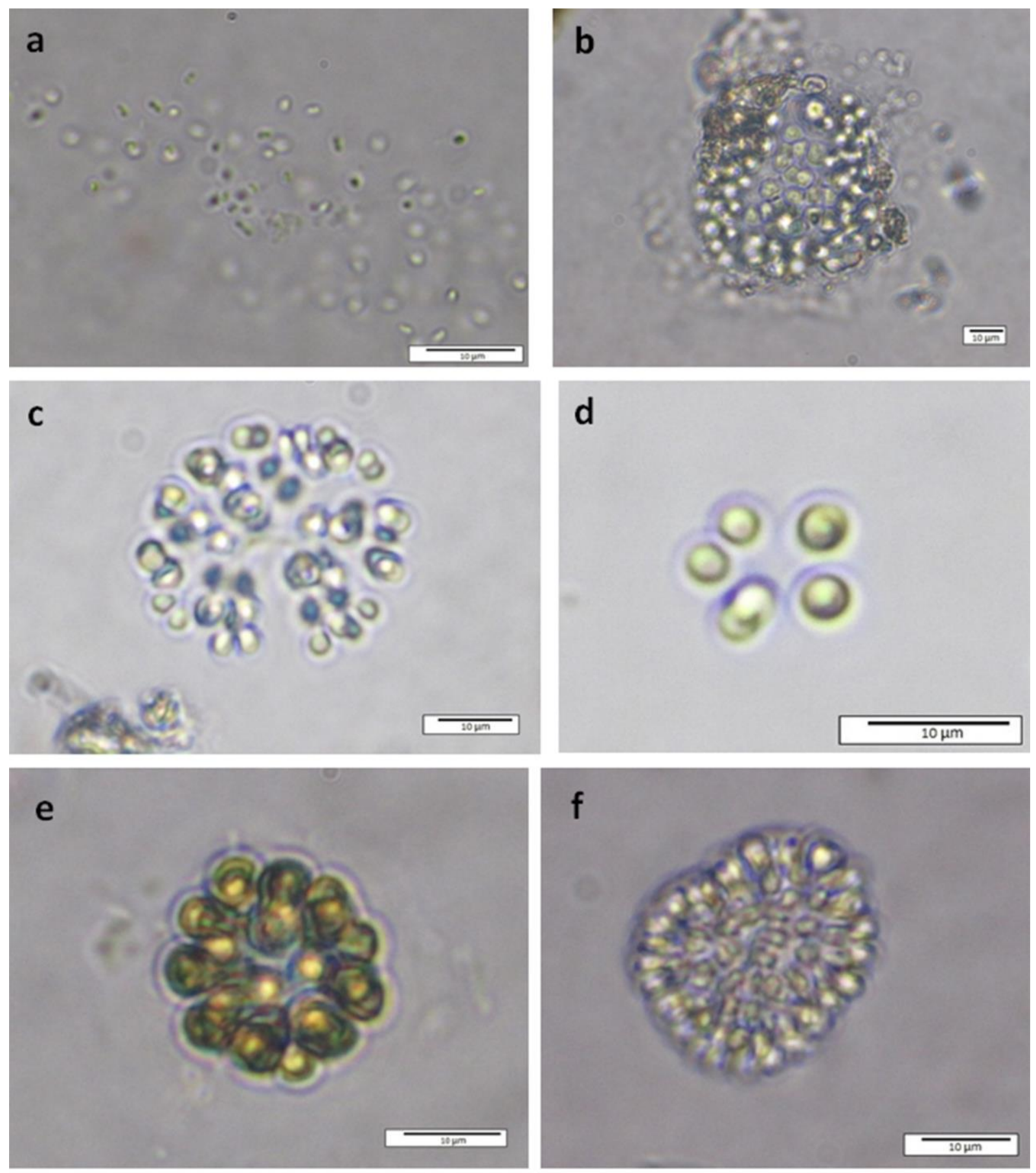

\section{f}
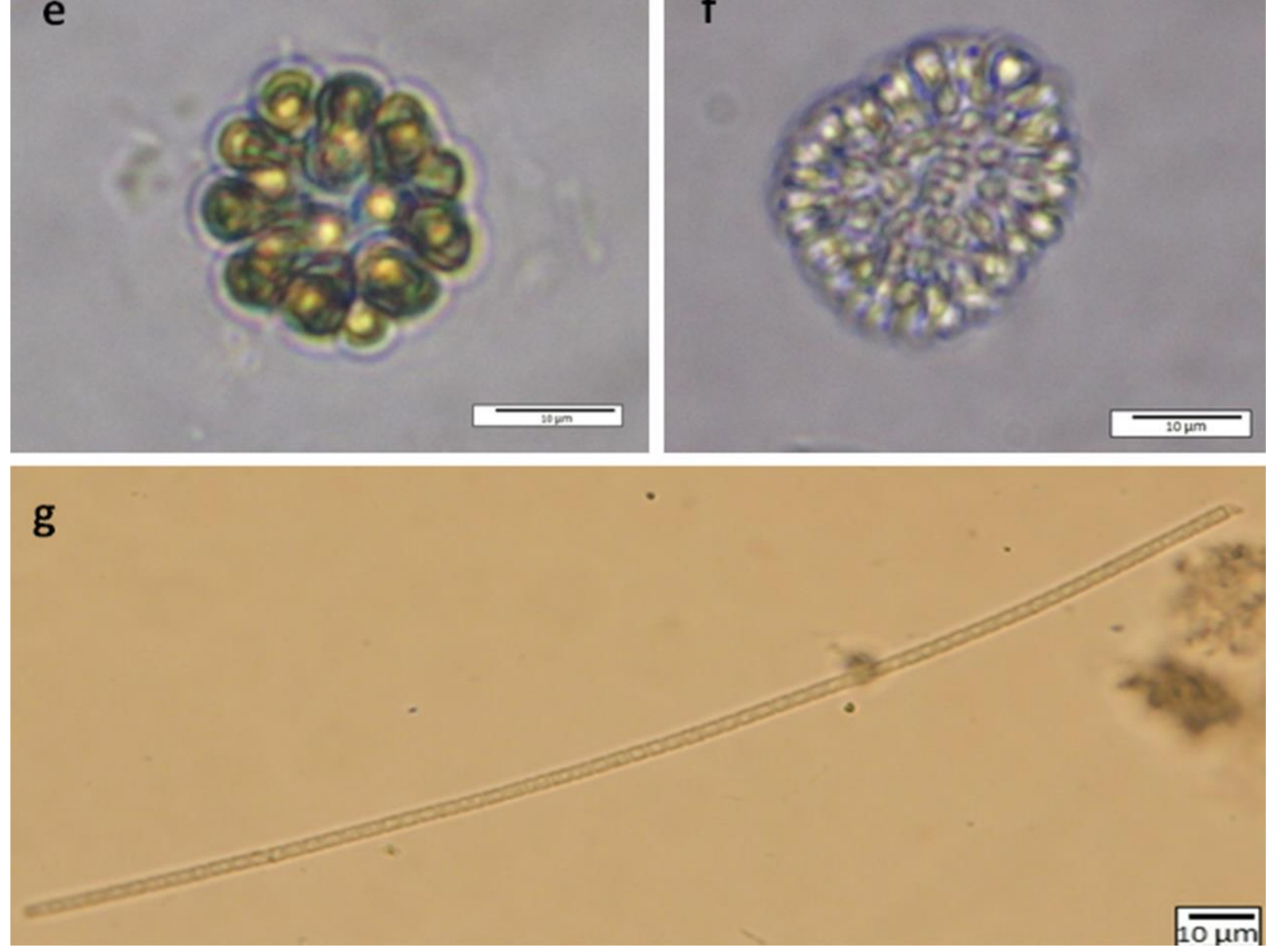

Figure 3. Cyanobacteria; a) Synechocystis parvula, b) Coelosphaerium aerugineum, c) Snowella fennica, d) Snowella litoralis, e) Woronichinia botrys, f) Woronichinia karelica, g) Leptolyngbya ectocarpi, (Scale $10 \mu \mathrm{m})$. 

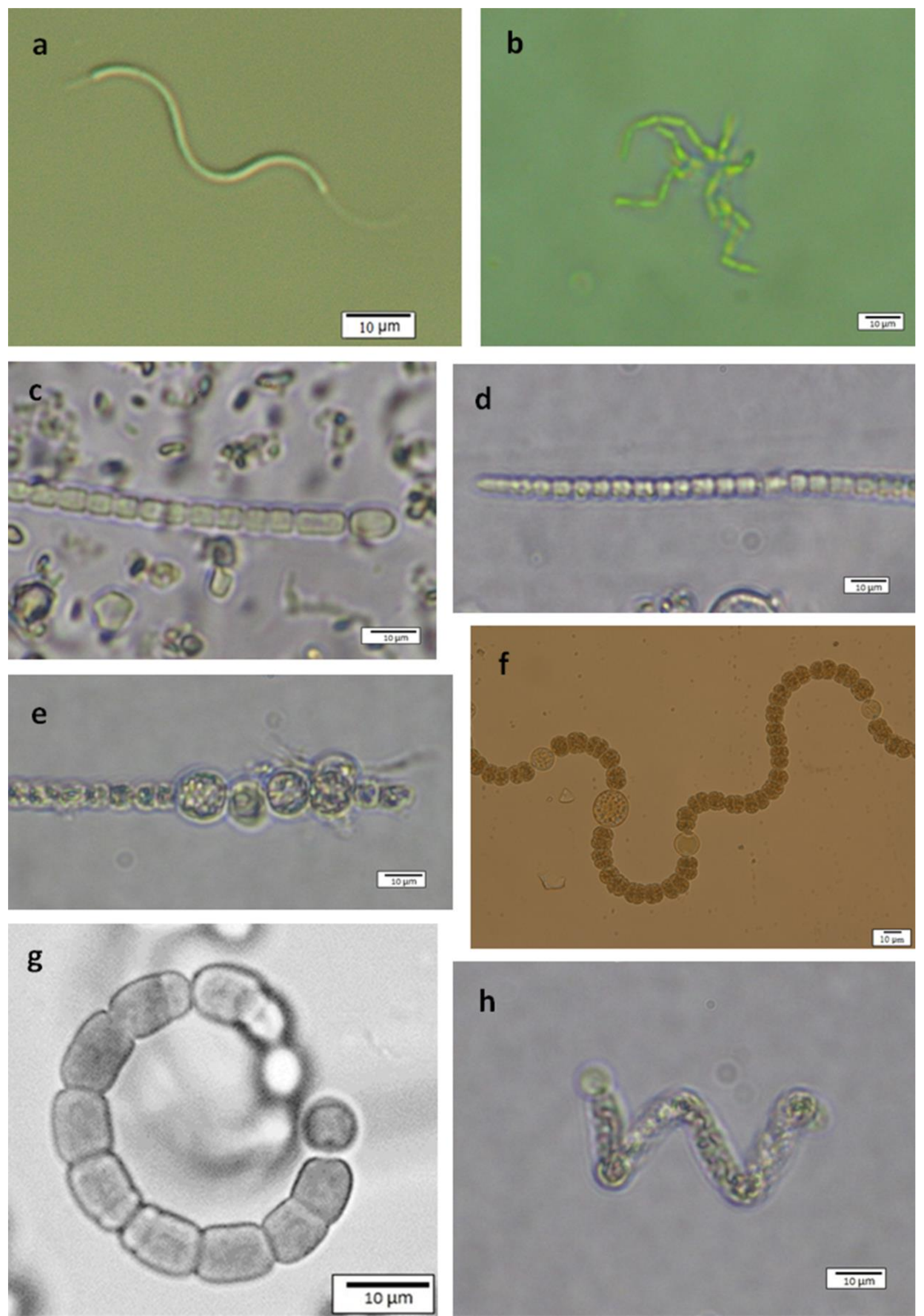

Figure 4. Cyanobacteria; a) Planktolyngbya microspira, b) Romeria gracilis, c) Anabaena laxa, d) Anabaena oblonga, e) Anabaena sphaerica var. attenuata, f) Nostoc caeruleum, g) Anabaenopsis milleri, h) Anabaenopsis rippkae, (Scale $10 \mu \mathrm{m})$. 

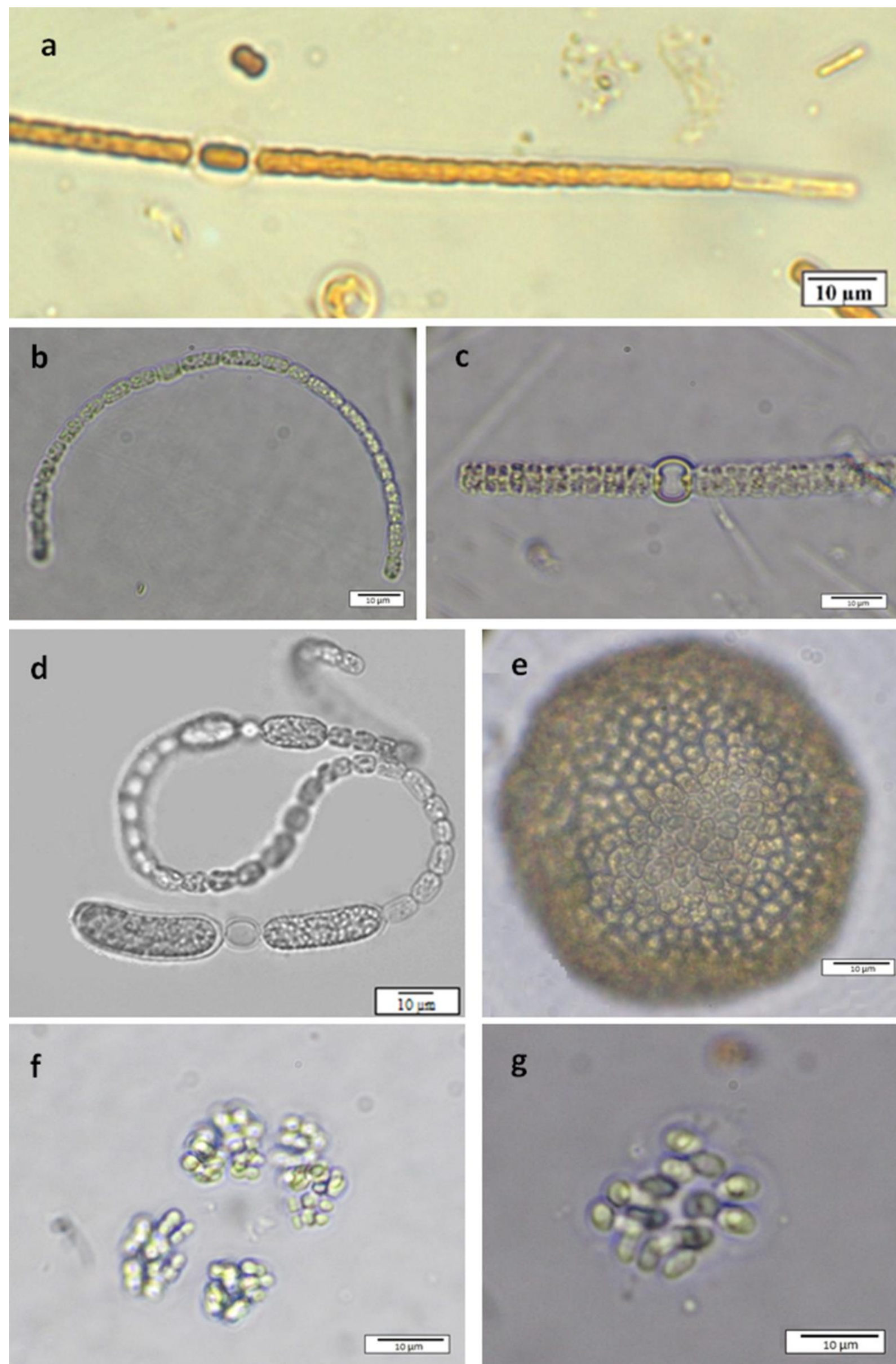

g

Figure 5. Cyanobacteria; a) Aphanizomenon klebahnii, b) Dolichospermum fallax, c) Nodularia sphaerocarpa, d) Dolichospermum lemmermannii, e) Pulvinularia suecica, f) Aphanothece atrocrustacea, g) Aphanothece comasii, (Scale $10 \mu \mathrm{m})$. 

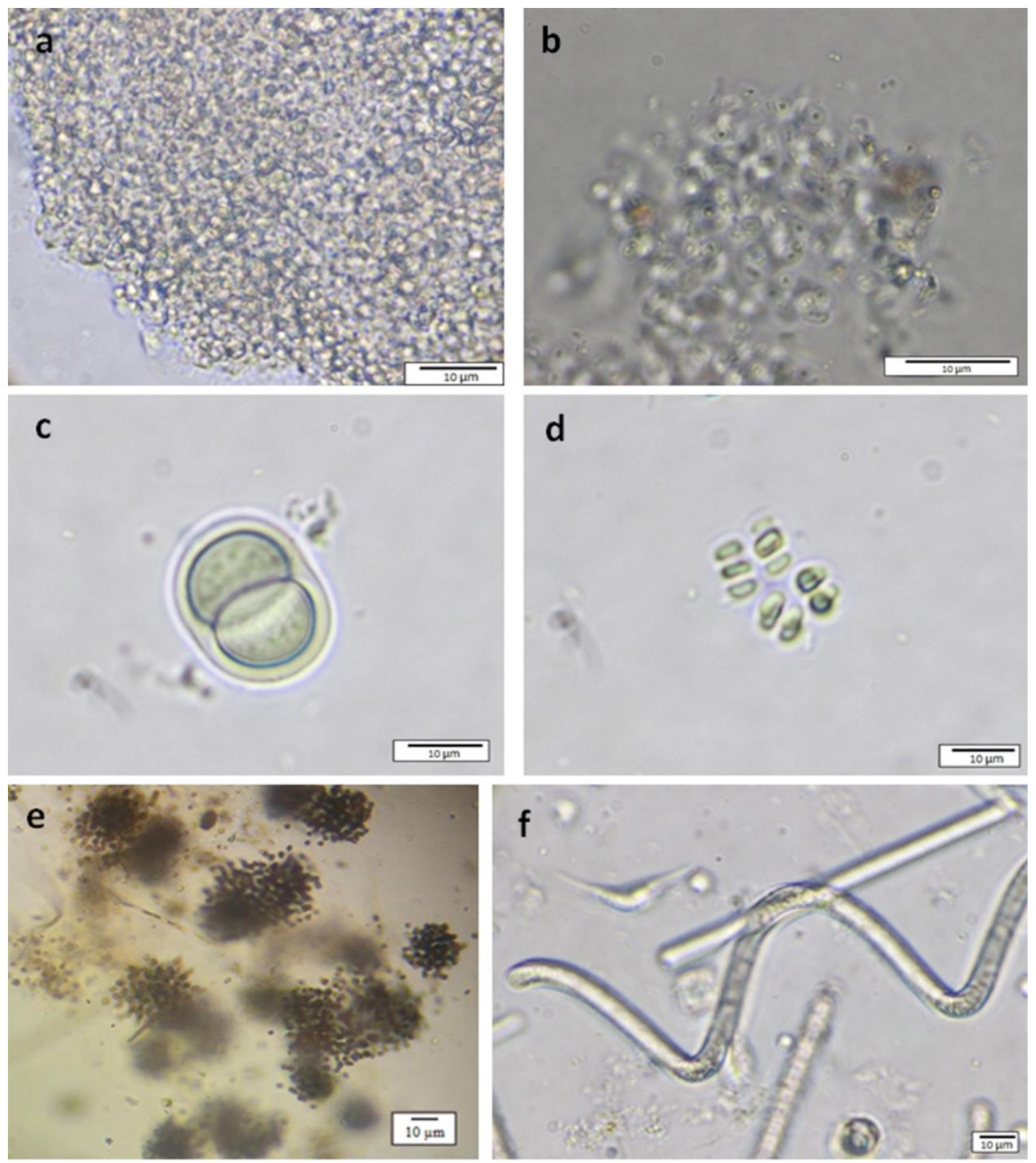

g

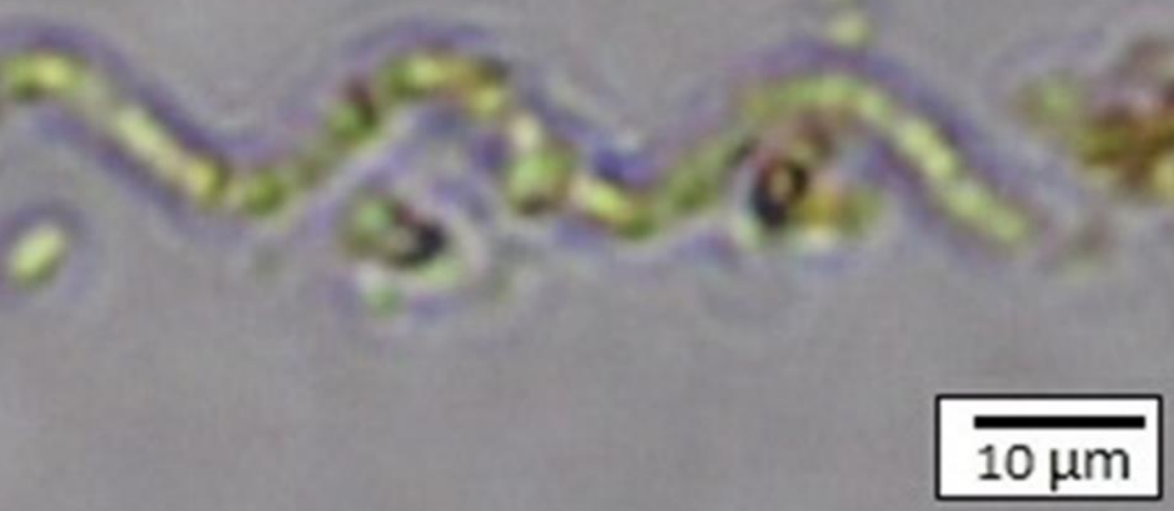

Figure 6. Cyanobacteria; a) Aphanothece floccosa, b) Gloeothece subtilis, c) Chroococcus lithophilus, d) Chroococcus mipitanensis, e) Microcystis botrys, f) Arthrospira platensis var. non-constricta, g) Limnospira fusiformis, (Scale $10 \mu \mathrm{m})$. 

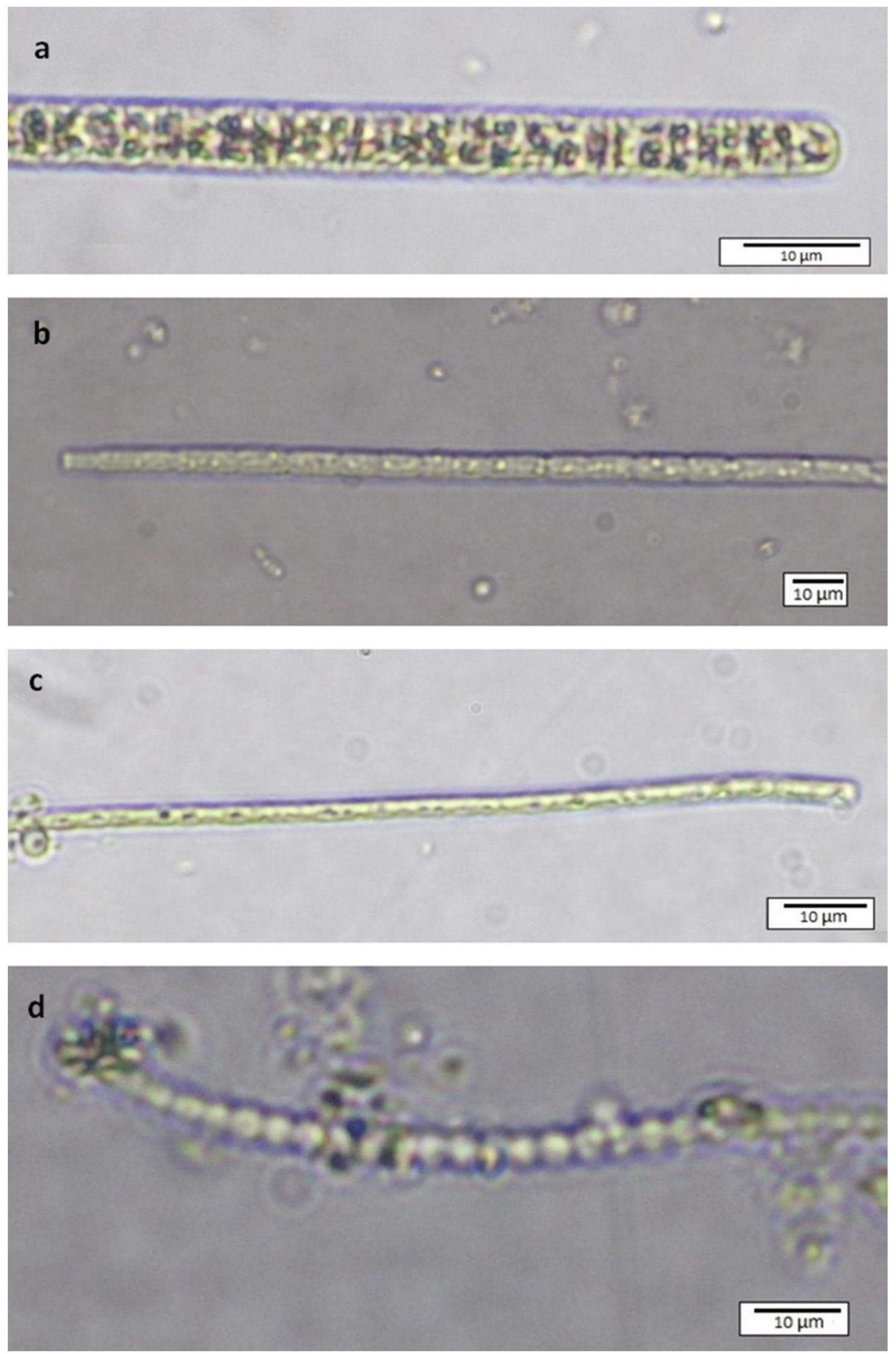

Figure 7. Cyanobacteria; a) Planktothrix isothrix, b) Oscillatoria chlorina f. perchlorina, c) Oscillatoria trichoides, d) Phormidium schultzii, (Scale $10 \mu \mathrm{m}$ ). 

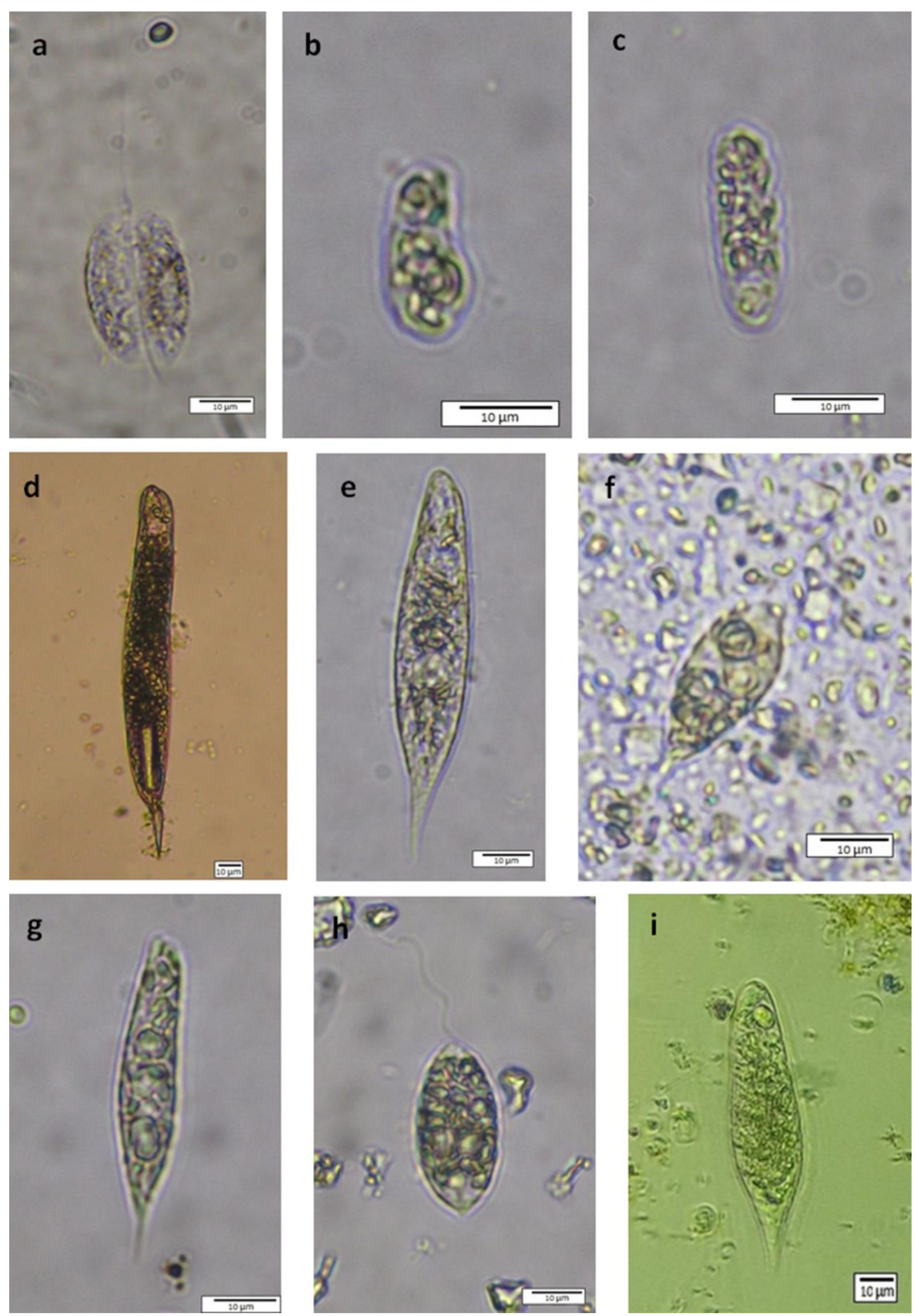

Figure 8. Euglenozoa; a) Anisonema prosgeobium, b) Astasia cylindrica, c) Astasia thiophila, d) Euglena antefossa, e) Euglena gracilis var. urophora, f) Euglena mainxii, g) Euglena rostrata, h) Euglena sacculiformis, i) Euglena velata, (Scale $10 \mu \mathrm{m})$. 

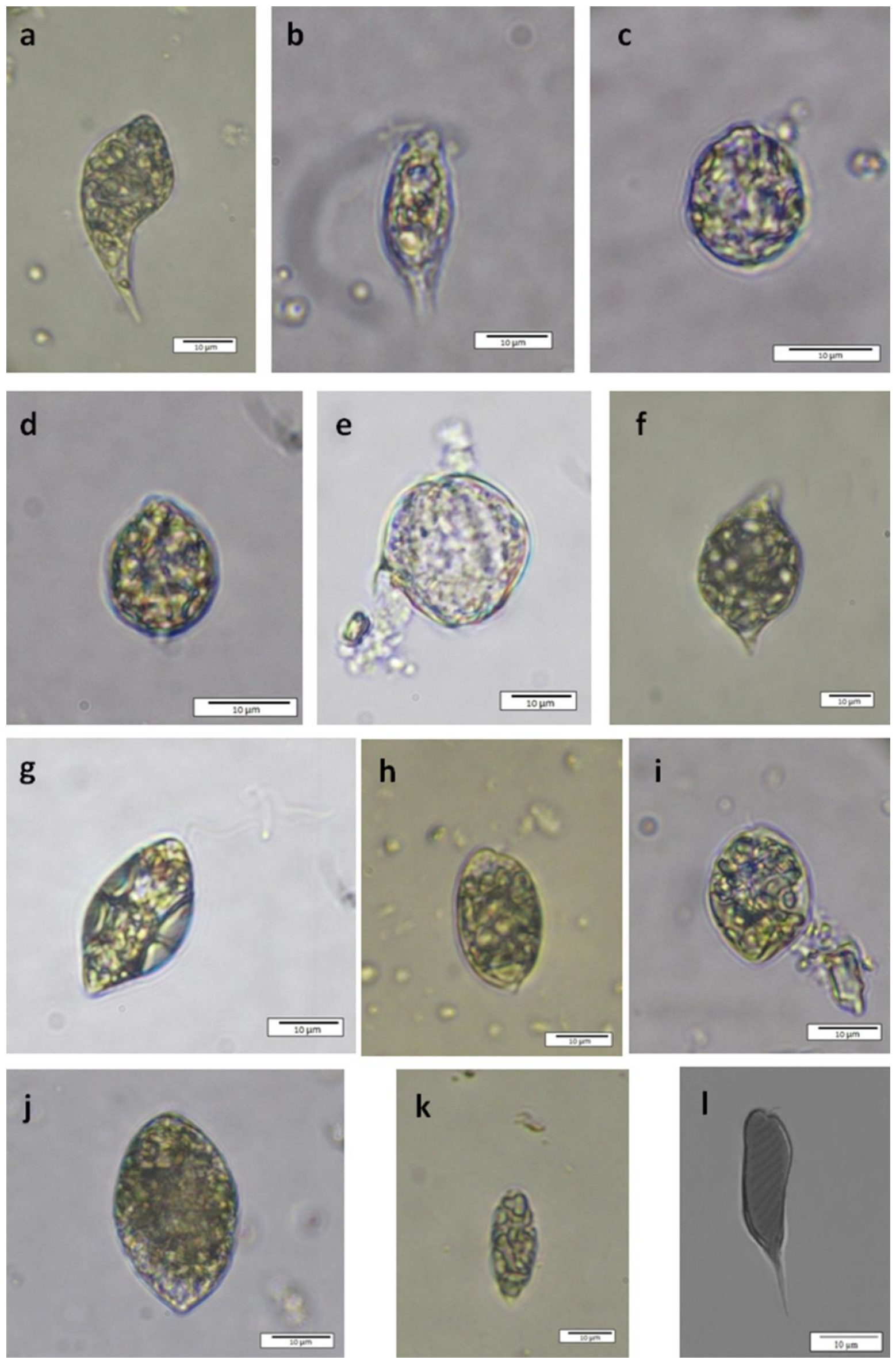

Figure 9. Euglenozoa; a) Euglenaria anabaena, b) Euglenopsis vacuolata, c) Lepocinclis conica, d) Lepocinclis fusiformis var. amphirhynchus, e) Lepocinclis lobata, f) Lepocinclis nayalii, g) Lepocinclis ovum var. angustatum, h) Lepocinclis ovum var. dimidiominor, i) Lepocinclis teres f. parvula, j) Lepocinclis texta var. mammillata, k) Menoidium semilunare var. regulare, l) Monomorphina aenigmatica, (Scale $10 \mu \mathrm{m})$. 

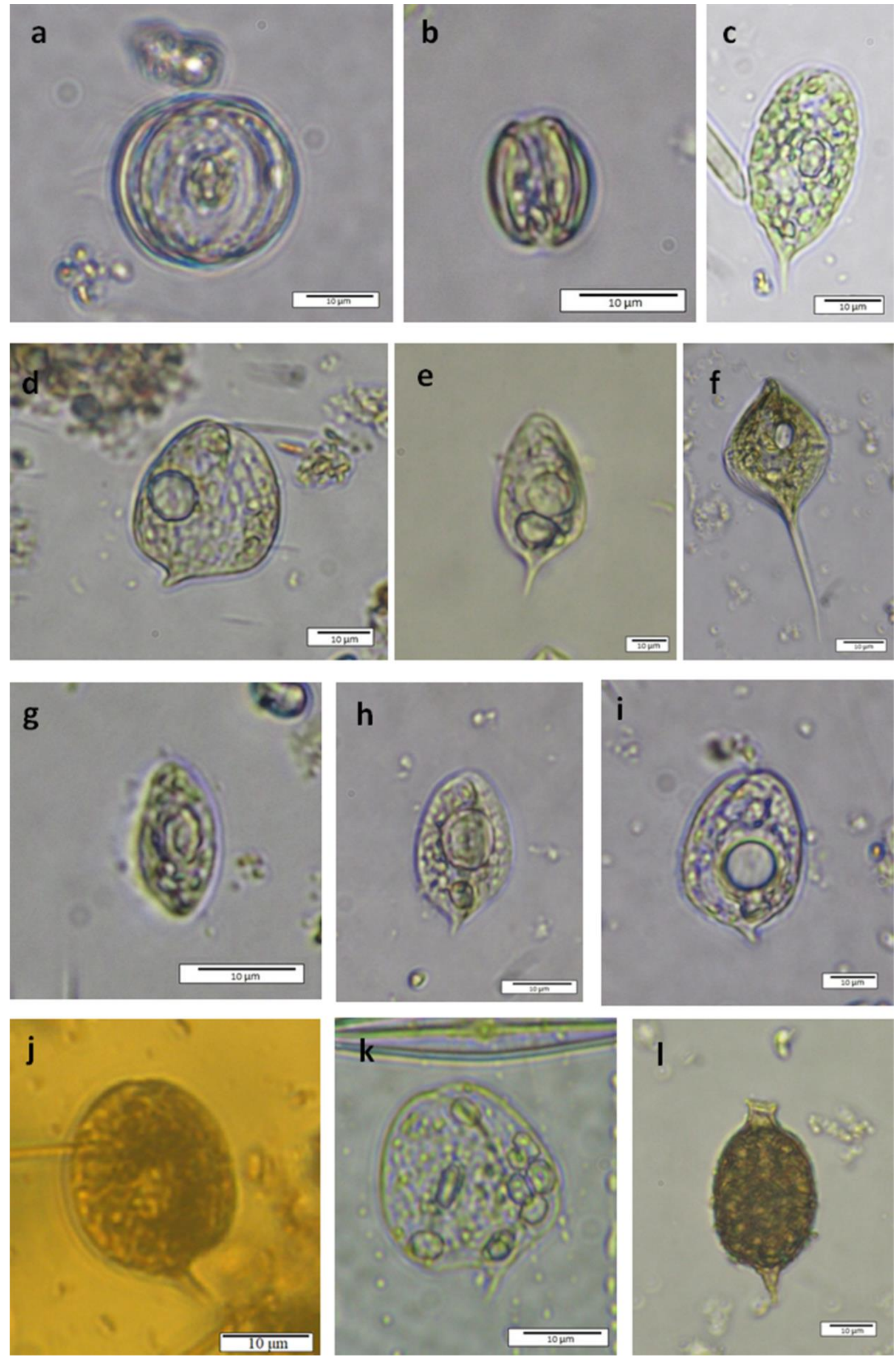

Figure 10. Euglenozoa; a) Petalomonas applanata, b) Phacus agilis var. inversus, c) Phacus applanatus, d) Phacus carinatus, e) Phacus caudatus, f) Phacus circumflexus, g) Phacus dangeardii, h) Phacus formosus, i) Phacus minutus, j) Phacus swirenkoi, k) Phacus tortuosus, I) Strombomonas acuminata var. amphora, (Scale $10 \mu \mathrm{m}$ ). 

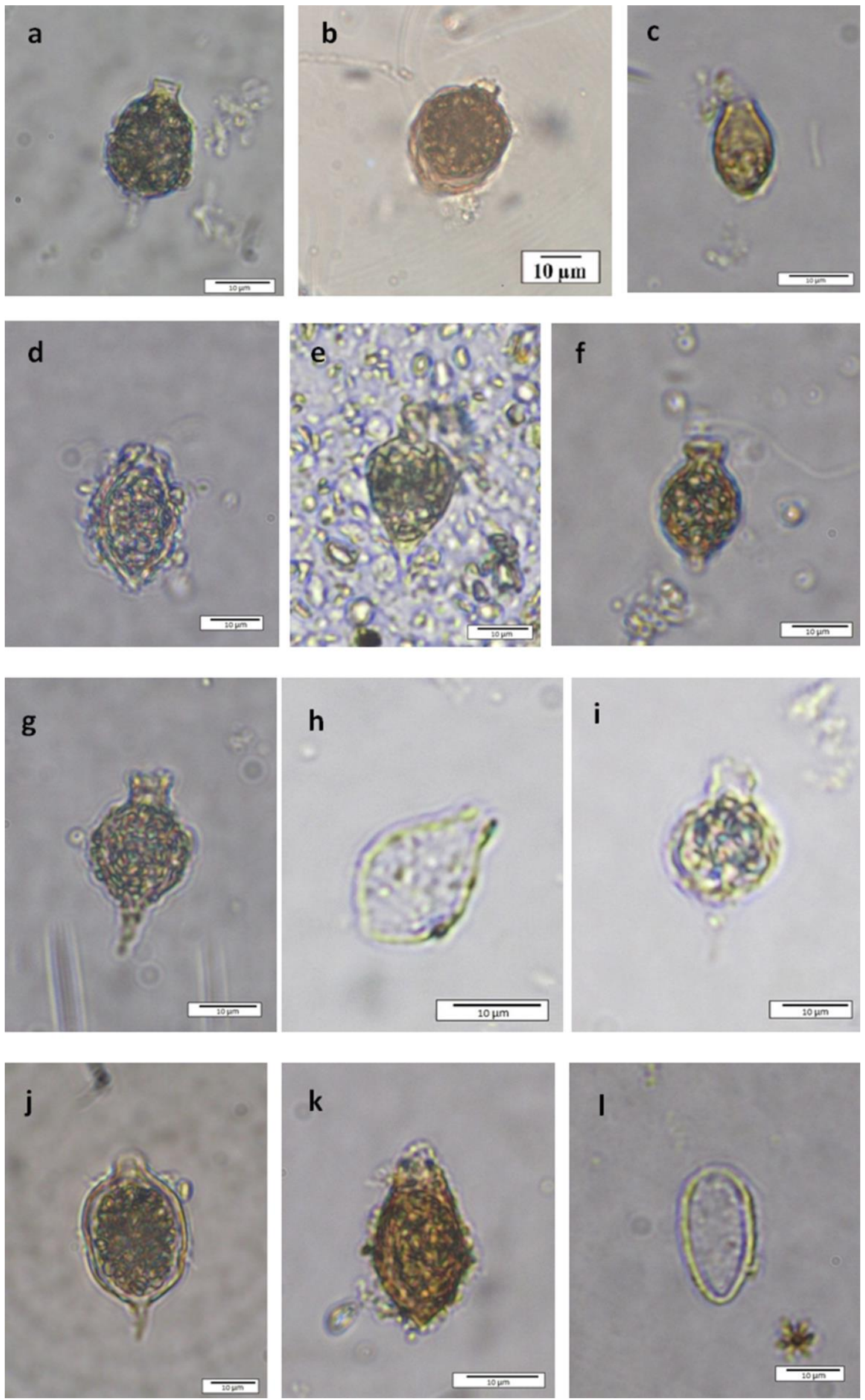

Figure 11. Euglenozoa; a) Strombomonas acuminata var. deflandreana, b) Strombomonas borystehniensis, c) Strombomonas inconstans, d) Strombomonas lanceolata, e) Strombomonas napiformis, f) Strombomonas praeliaris var. nana, g) Strombomonas rotunda f. hortobagyi, h) Strombomonas subcurvata var. africana, i) Strombomonas treubii, j) Strombomonas urceolata, k) Strombomonas aspera, I) Trachelomonas abrupta f. angustata, (Scale $10 \mu \mathrm{m}$ ). 

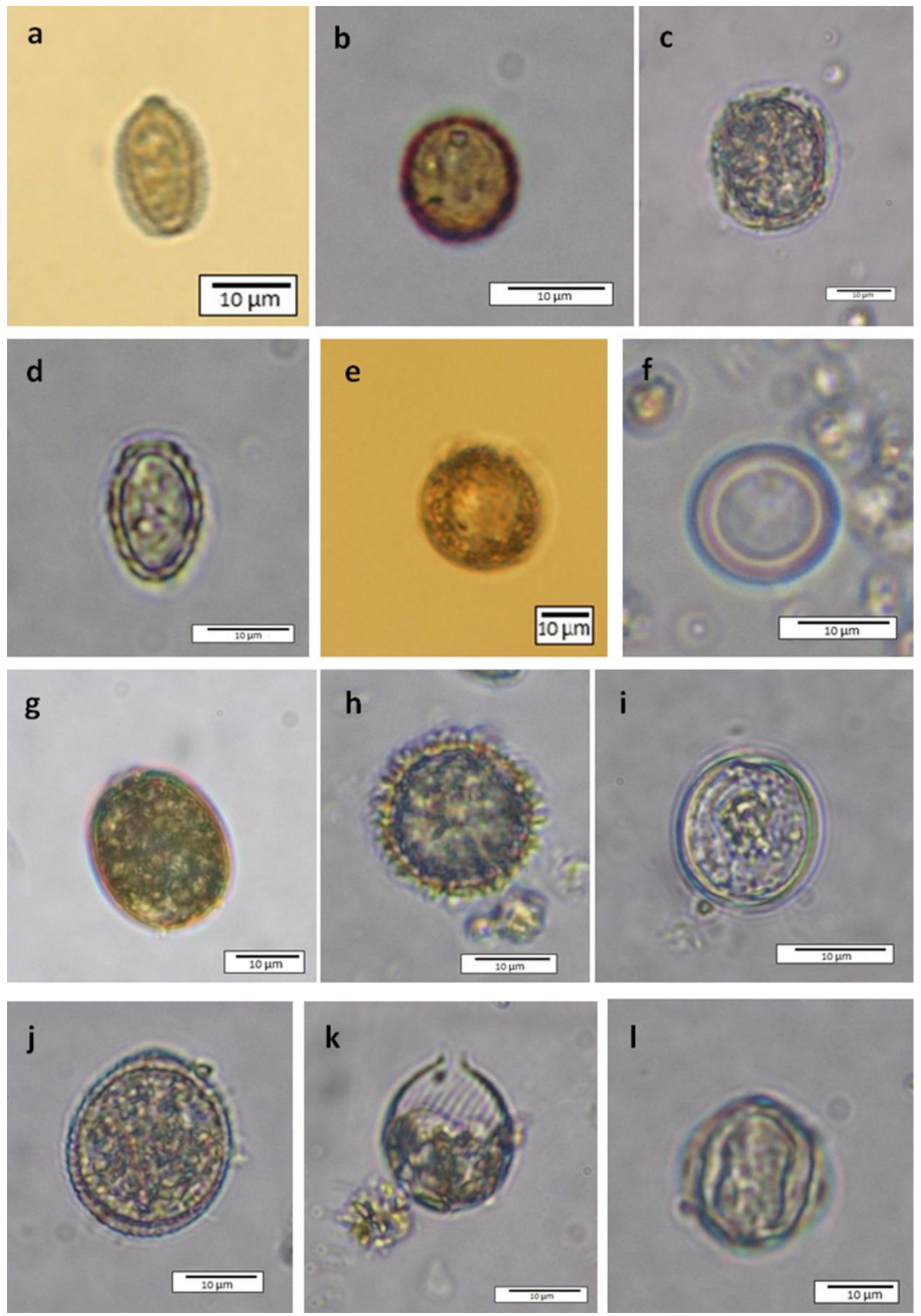

Figure 12. Euglenozoa; a) Trachelomonas amphora, b) Trachelomonas anulifera, c) Trachelomonas bacillifera f. sparsispina, d) Trachelomonas chodati, e) Trachelomonas columba, f) Trachelomonas curta var. minima, g) Trachelomonas dangeardii var. glabra, h) Trachelomonas globularis var. boyeri, i) Trachelomonas grandis, j) Trachelomonas granulosa var. subglobosa, k) Trachelomonas heduma, I) Trachelomonas hexangulata var. hexagona, (Scale $10 \mu \mathrm{m}$ ) 

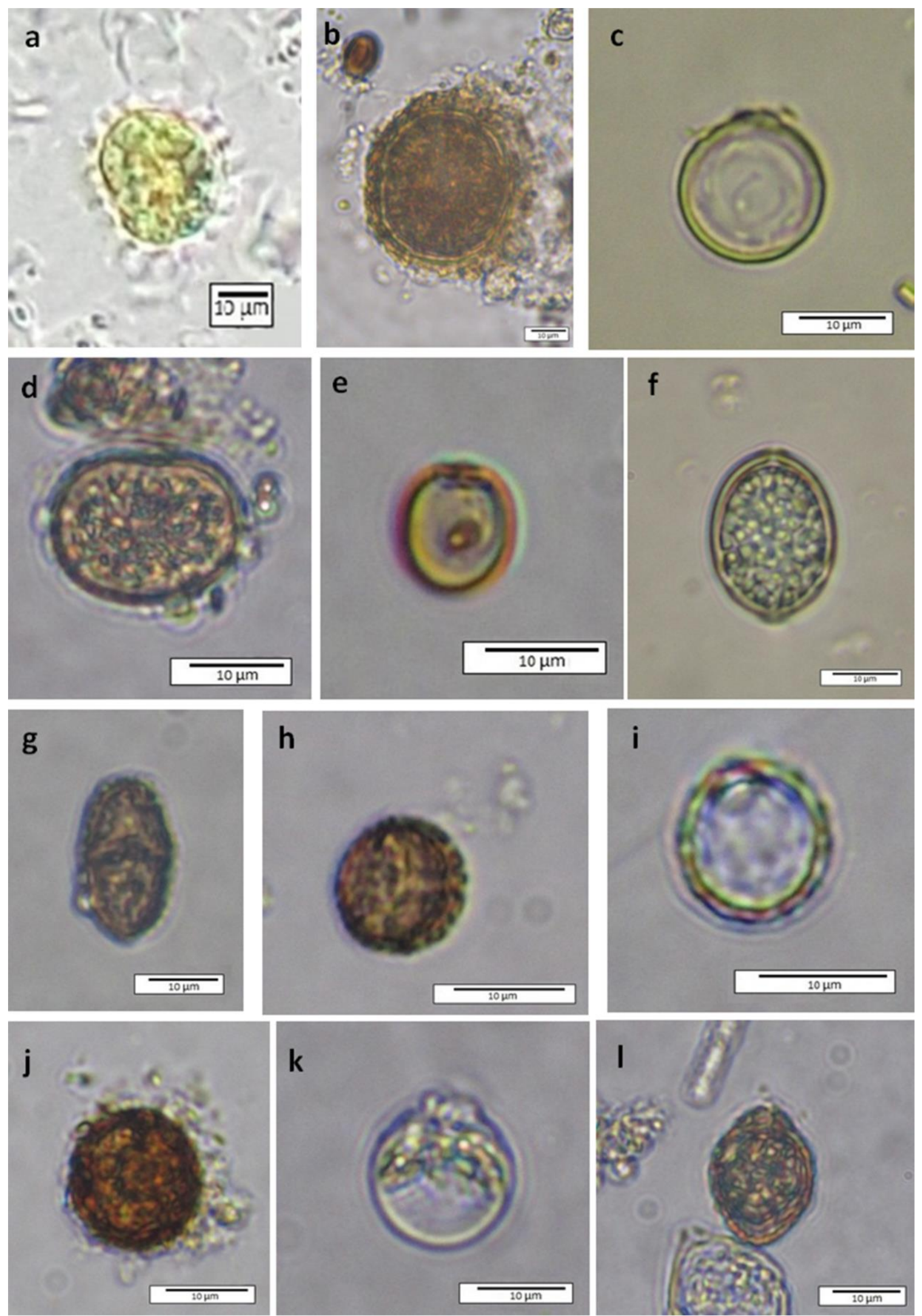

Figure 13. Euglenozoa; a) Trachelomonas horrida, b) Trachelomonas kelloggii var. nana, c) Trachelomonas komarowii, d) Trachelomonas lismorensis var. inermis, e) Trachelomonas oblonga var. australica, f) Trachelomonas obovata var. klebsiana, g) Trachelomonas orenburgika var. ornata, h) Trachelomonas perlata, i) Trachelomonas pseudofelix, j) Trachelomonas stokesiana f. meandrina, k) Trachelomonas rugulosa var. obliqua, l) Trachelomonas scabra var. ovata f. minör, (Scale $10 \mu \mathrm{m})$. 

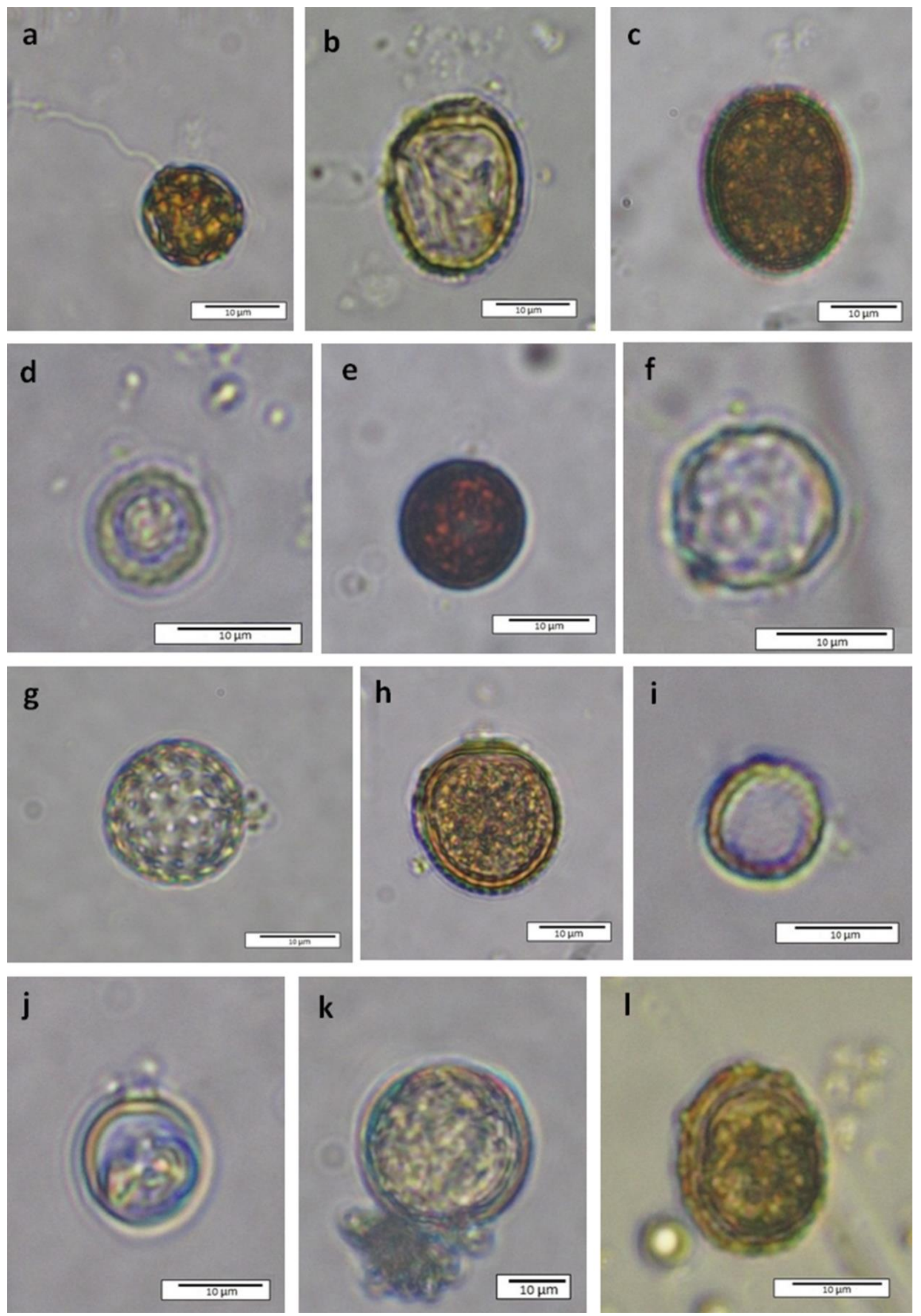

Figure 14. Euglenozoa; a) Trachelomonas scabra var. coberensis, b) Trachelomonas sydneyensis var. minima, c) Trachelomonas sydneyensis var. obesa, d) Trachelomonas tuberculata, e) Trachelomonas varians f. globosa, f) Trachelomonas verrucosa $\mathrm{f}$. irregularis, g) Trachelomonas verrucosa f. sparseornata, h) Trachelomonas verrucosa var. macrotuberculata, i) Trachelomonas verrucosa var. spirogyra, j) Trachelomonas volvocina var. derephora, k) Trachelomonas volvocinopsis var. khannae, I) Trachelomonas zorensis, (Scale $10 \mu \mathrm{m})$. 

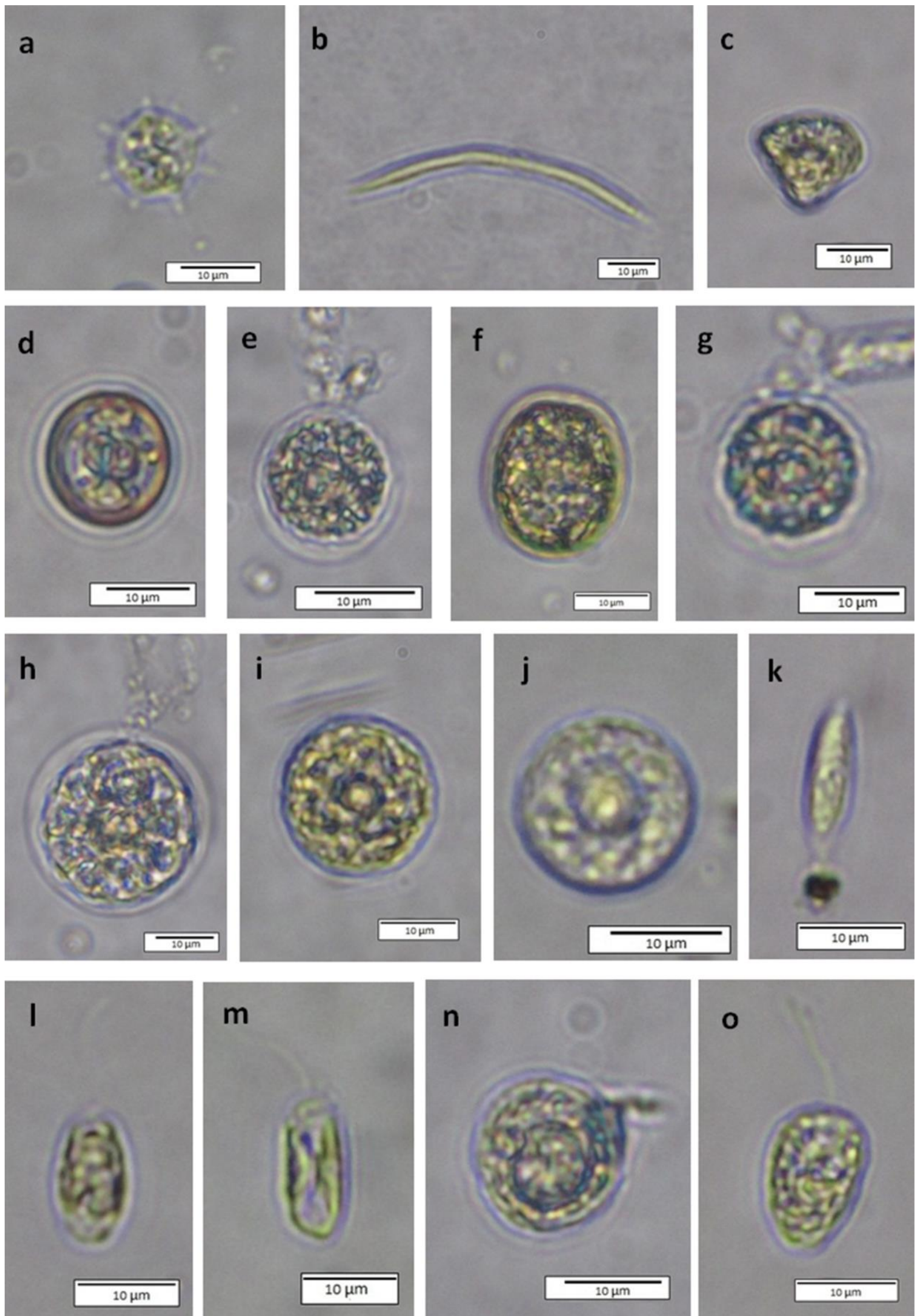

Figure 15. Chlorophyta; a) Acanthosphaera zachariasii, b) Ankistrodesmus stipitatus, c) Aulacomonas submarina, d) Carteria agloeformis, e) Carteria fritschii, f) Carteria huberi, g) Carteria inversa, h) Carteria lohammari, i) Carteria sphaerica, j) Carteria stellifera, k) Characium angustum, I) Chlamydomonas anglica, m) Chlamydomonas bichlora, n) Chlamydomonas confinis, o) Chlamydomonas conocylindrus, (Scale $10 \mu \mathrm{m}$ ). 

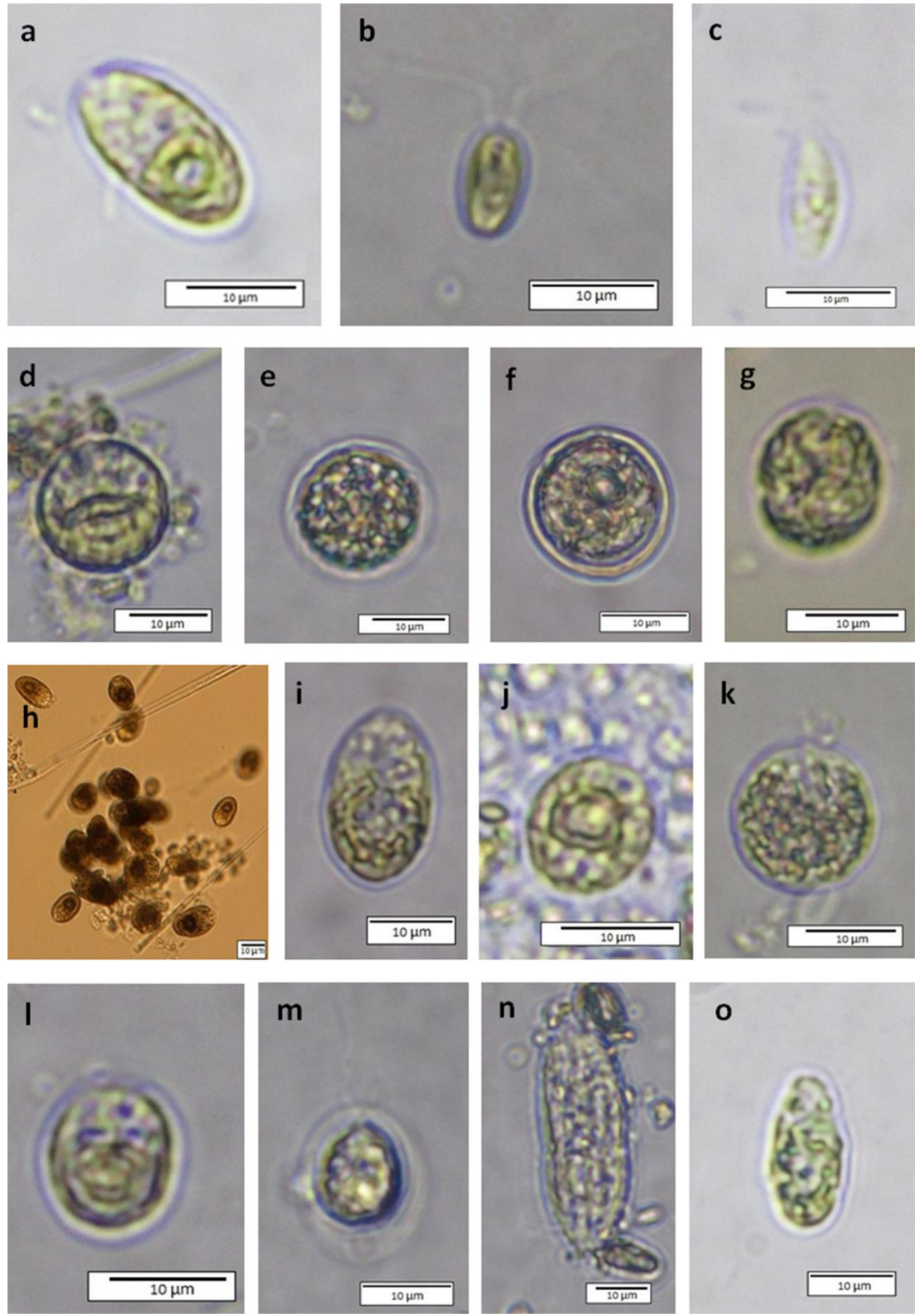

Figure 16. Chlorophyta; a) Chlamydomonas crassa, b) Chlamydomonas elegans, c) Chlamydomonas gloeophila, d) Chlamydomonas granulosa, e) Chlamydomonas incerta, f) Chlamydomonas incisa, g) Chlamydomonas klinobasis, h) Chlamydomonas lapponica, i) Chlamydomonas macroplastida, j) Chlamydomonas macropyrenoidosa, k) Chlamydomonas metapyrenigera, I) Chlamydomonas microsphaerella, m) Chlamydomonas nivalis, n) Chlamydomonas penium, o) Chlamydomonas pulvinata, (Scale $10 \mu \mathrm{m})$. 

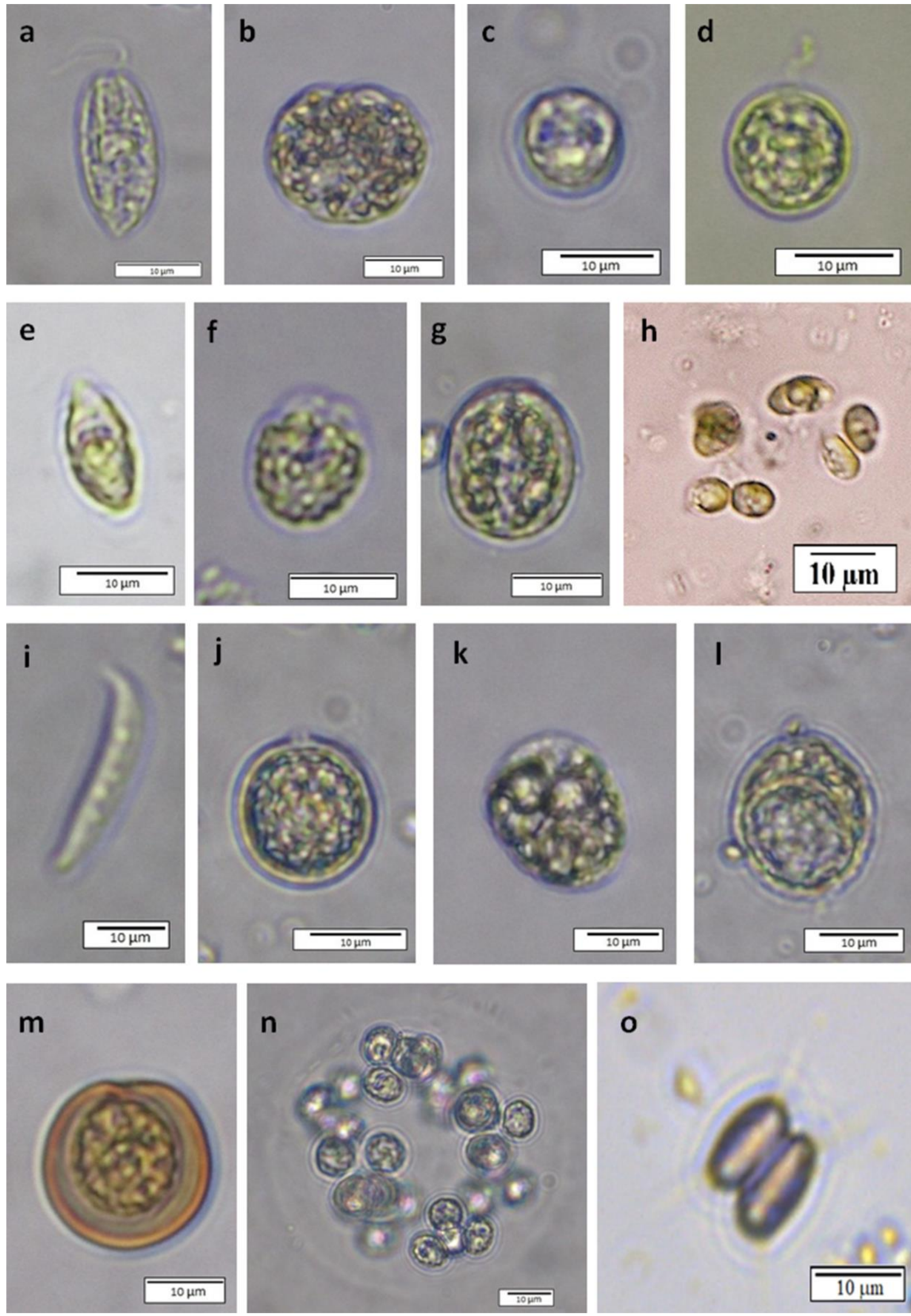

Figure 17. Chlorophyta; a) Chlamydomonas rhopaloides, b) Chlamydomonas rotifera, c) Chlamydomonas simplex, d) Chlamydomonas skujae, e) Chlamydomonas tremulans, f) Chlamydomonas truncata, g) Chlamydomonas upsaliensis, h) Chlorella chlorelloides, i) Chlorolobion lunulatum, j) Chloromonas vernalis, k) Chloromonas vesterbottnica, I) Chloromonas westiana, m) Coccomonas platyformis, n) Coenococcus planctonicus, o) Desmodesmus abundans var. brevicauda, (Scale $10 \mu \mathrm{m})$. 

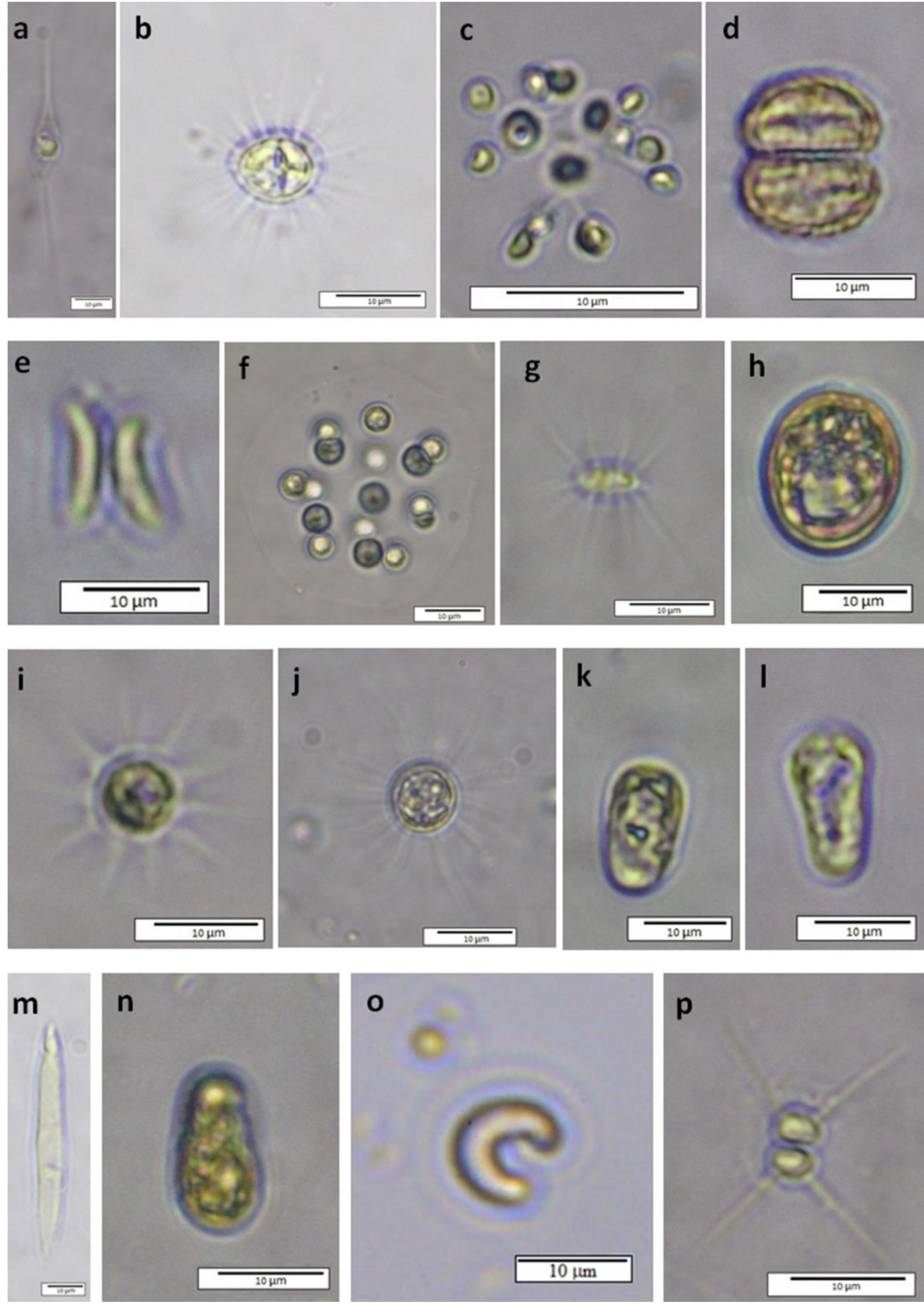

Figure 18. Chlorophyta; a) Desmatractum indutum b) Dicellula geminata, c) Dictyosphaerium subsolitarium, d) Didymocystis inermis, e) Didymogenes palatina, f) Eutetramorus tetrasporus, g) Franceia javanica, h) Gloeomonas tecta, i) Golenkinia brevispina, j) Golenkinia maxima, k) Hafniomonas montana, I) Hafniomonas reticulata, m) Hyalogonium elangatum, n) Ixipapillifera sacculiformis, o) Kirchneriella major, p) Micractinium elongatum, (Scale $10 \mu \mathrm{m})$. 

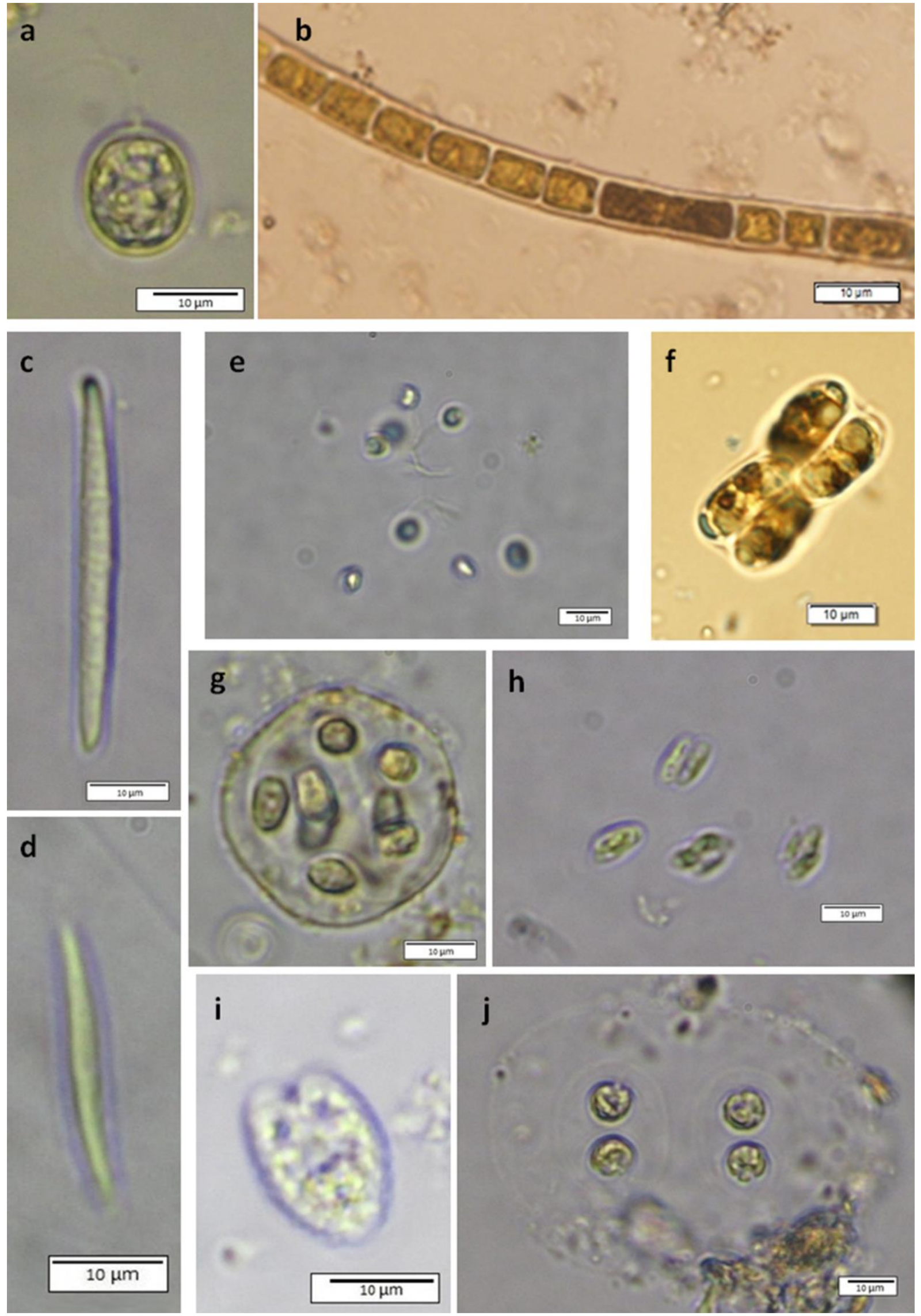

Figure 19. Chlorophyta; a) Microglena coccifera, b) Microspora amoena var. gracilis, c) Monoraphidium obtusum, d) Monoraphidium pseudobraunii, e) Mucidosphaerium sphagnale, f) Oocystis tainoensis, g) Palmococcus hercynicus, h) Palmococcus reniformis, i) Papenfussiomonas cordata, j) Paulschulzia pseudovolvox, (Scale $10 \mu \mathrm{m})$. 

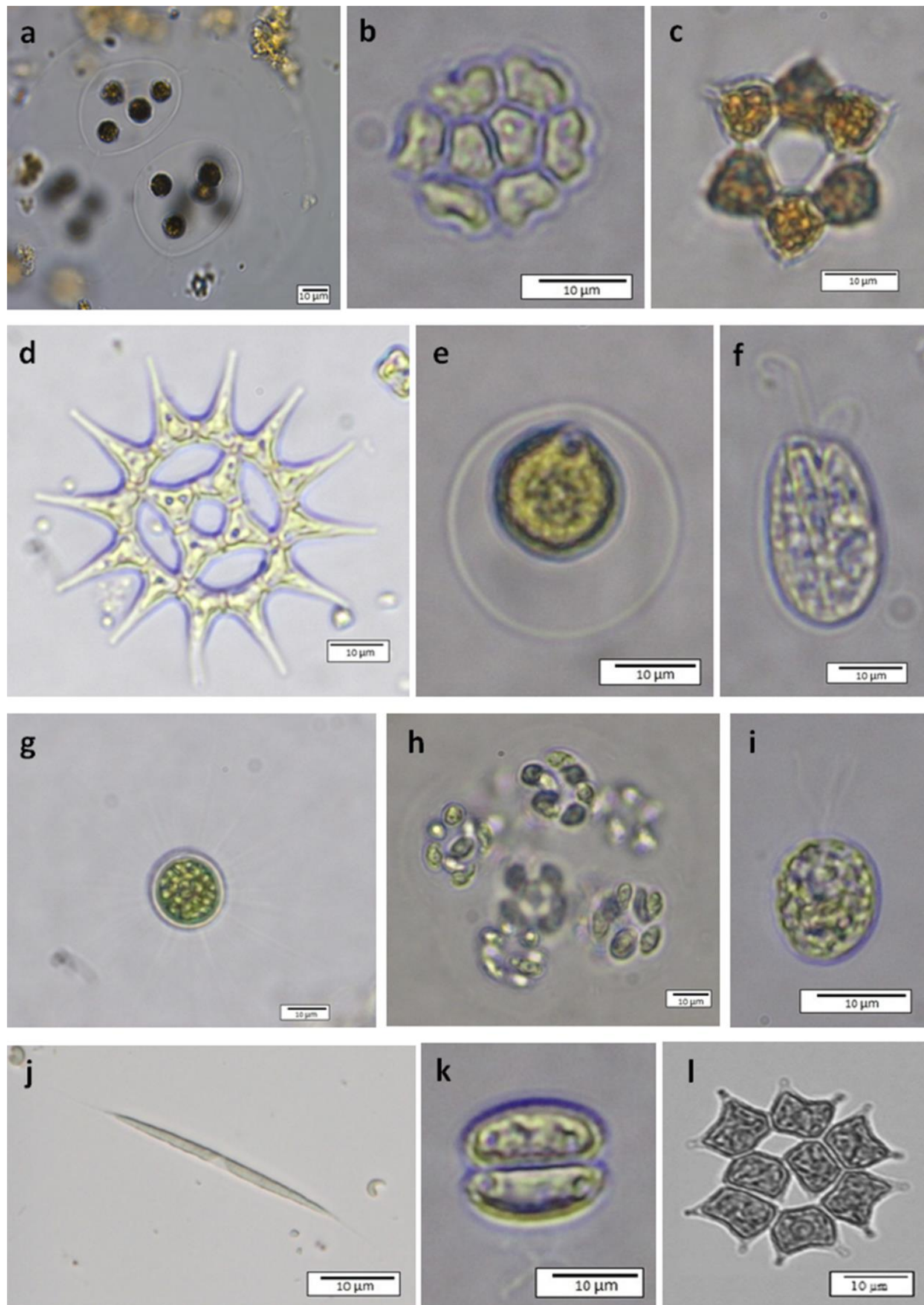

Figure 20. Chlorophyta; a) Paulschulzia tenera, b) Pediastrum orbitale, c) Pediastrum ovatum, d) Pediastrum simplex var. clathratum, e) Phacotus glaber, f) Phyllariochloris caeca, g) Golenkinia viridis, h) Planktococcomyxa lacustris, i) Platymonas cordiformis, j) Podohedriella falcata, k) Pseudodidymocystis fina, I) Pseudopediastrum subgranulatum, (Scale $10 \mu \mathrm{m})$. 

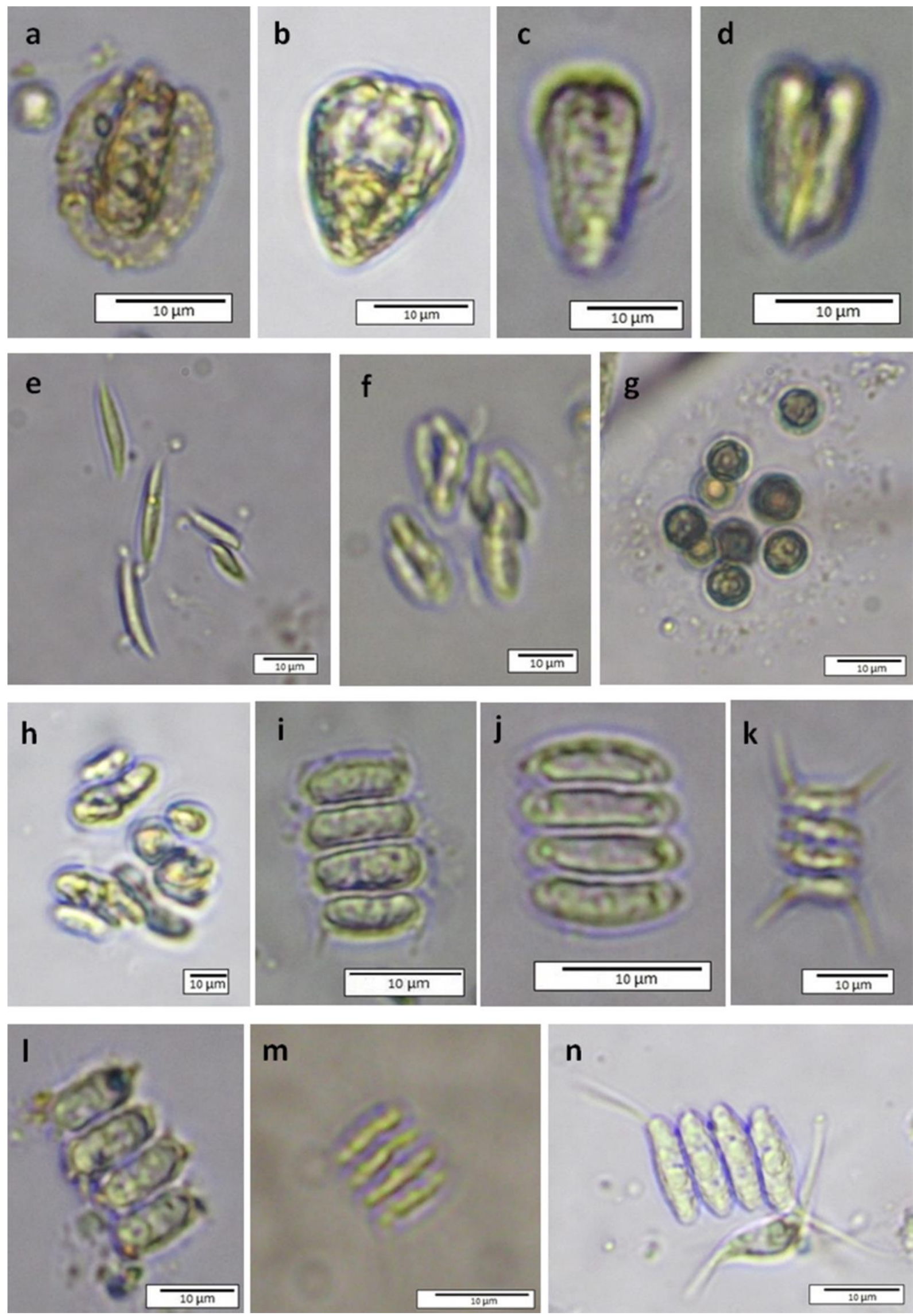

Figure 21. Chlorophyta; a) Pteromonas rugosa, b) Pyramimonas delicatula, c) Pyramimonas inconstans, d) Pyramimonas splendidissima, e) Quadrigula chodatii, f) Quadrigula sabulosa, g) Radiococcus bavaricus, h) Rayssiella hemisphaerica, i) Scenedesmus aldavei, j) Scenedesmus calyptratus, k) Scenedesmus formidolosus, I) Scenedesmus fusiformis, m) Scenedesmus gutwinskii var. bacsensis, n) Scenedesmus opoliensis var. bicaudatus, (Scale $10 \mu \mathrm{m})$. 

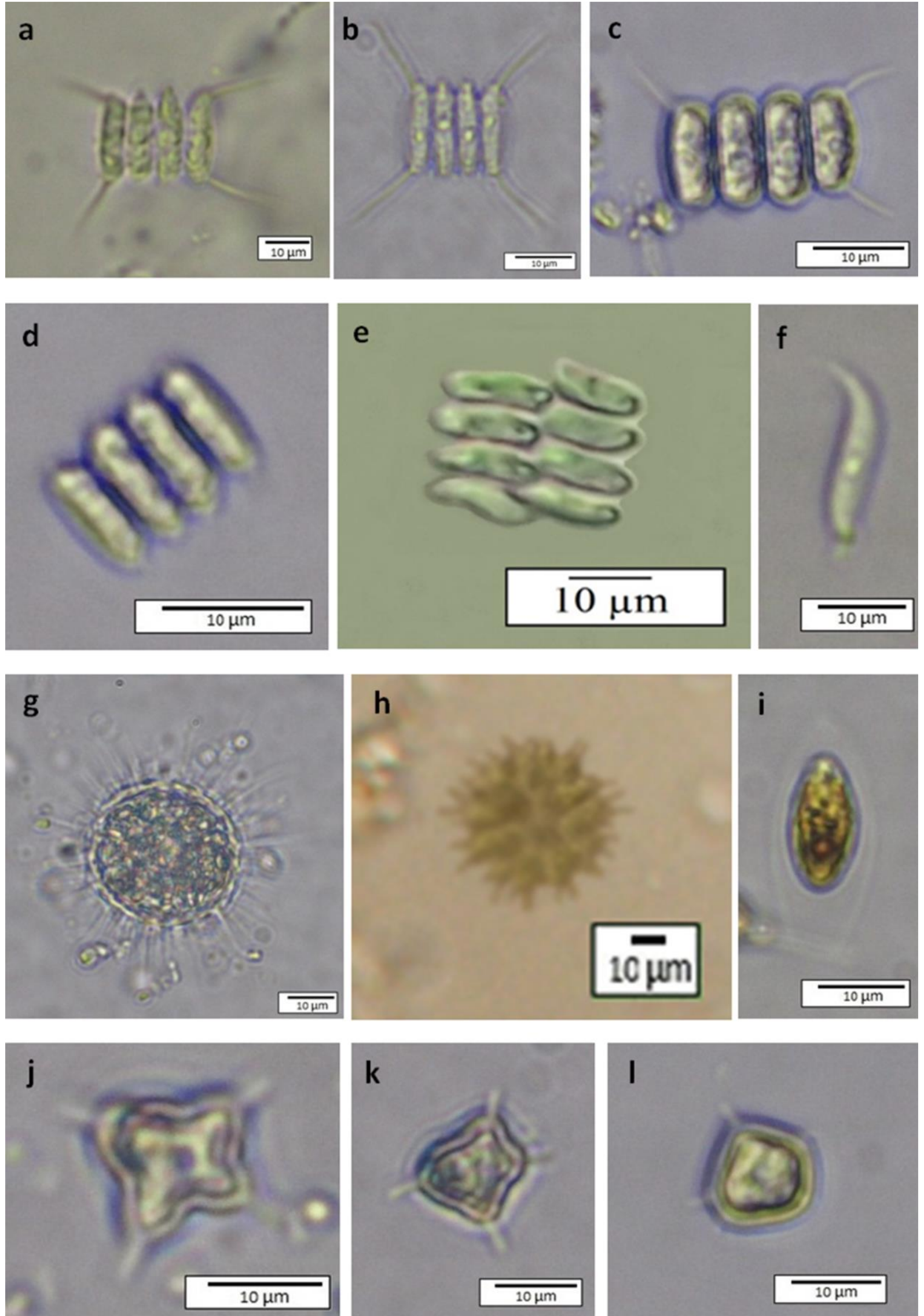

Figure 22. Chlorophyta; a) Scenedesmus protuberans var. minor, b) Scenedesmus protuberans f. danubianus, c) Scenedesmus pseudoquadricauda, d) Scenedesmus similagineus, e) Scenedesmus tibiscensis, f) Schroederia ecsediensis, g) Siderocystopsis punctifera, h) Sorastrum spinulosum, i) Sphaerellopsis agloë, j) Tetraëdron minimum f. apiculatum, k) Tetraëdron octaedricum, I) Tetraëdron octaedricum var. spinosum, (Scale $10 \mu \mathrm{m})$. 

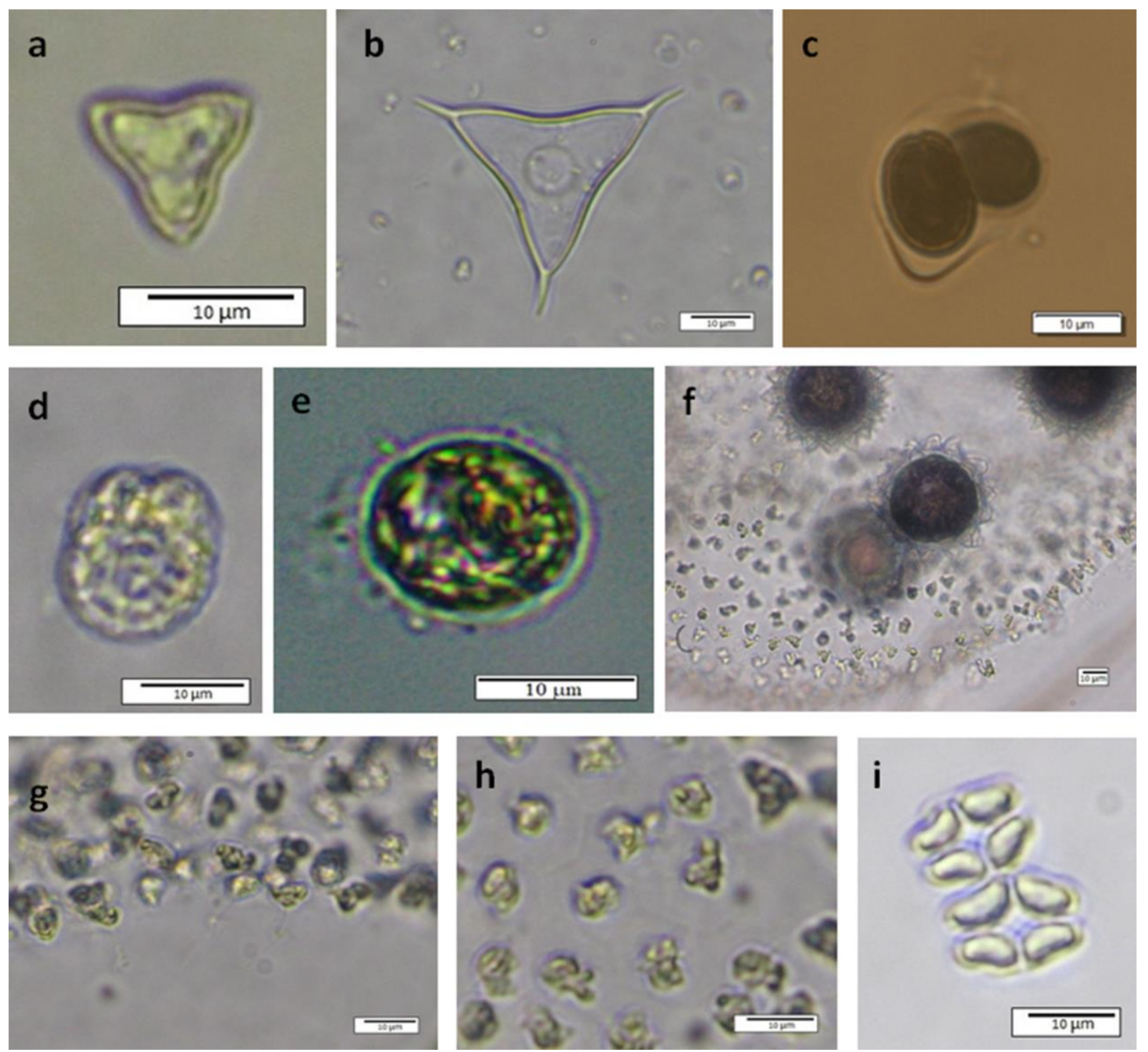

Figure 23. Chlorophyta; a) Tetraëdron trigonum f. minus-obtusum, b) Tetraëdron trigonum var. longispinum, c) Tetraselmis arnoldii, d) Tetraselmis elliptica, e) Trochiscia granulata, f-g-h) Volvox rousseletii, i) Willea crucifera, (Scale $10 \mu \mathrm{m}$ ).

these basins are wilderness with extreme climatic conditions. When we analyze the relationship between the number of lakes sampled in 25 basins and the number of new records, it is seen that the most remarkable basins are Asi, Western Mediterranean, Kızılırmak and Ceyhan. Although the number of studied lakes (13 and 8, respectively) in the Western Mediterranean and Asi basins was low, the number of new records ( 43 and 38 , respectively) were found to be quite high compared to other basins. The number of studied lakes is high in Kızılırmak, Ceyhan, Western Black Sea, Yeşilırmak, and Büyük Menderes basins, but hardly any of new records have been detected. This indicates that the lake areas in these basins that we have sampled are not wilderness.

In all three divisions, it was observed that tolerant species are more dominant in the basins among the species with rare and common distribution areas. This shows that the water quality level of the water resources in the basins is not very good. The fact that the majority of both tolerant and sensitive species in all three divisions were detected in the Sakarya and FiratDicle basins shows that the biodiversity is relatively high in both basins.

\section{Conclusion}

The Establishment of Reference Monitoring Network in Turkey Project, which is coordinated by the Ministry of Agriculture and Forestry, is the most comprehensive research project on the ecology of wetlands in Turkey in recent years. 1363 phytoplankton species and sub-species are determined from the 275 lakes. 238 taxa of them in three divisions are new records for the algal flora of Turkey. The present study includes the new records from the three main algal divisions, expected to contribute to the Algal database of Turkey (http://turkiyealgleri.hitit.edu.tr) edited by Maraşlıoğlu and Gönülol (2021) and to the Turkey Algae list published by Taşkın et al. (2019). 


\section{Ethical Statement}

Not applicable

\section{Funding Information}

This study was supported by the Ministry of Agriculture and Forestry, Directorate General of Water Management (Project number: 2011K050400).

\section{Author Contribution}

First Author: Conceptualization, Writing-review, editing, Data Curation, Formal Analysis, Investigation, Methodology, Visualization

Second Author: Data Curation, Formal Analysis, Investigation, Methodology, Visualization

Third Author: Conceptualization, Data Curation, Formal Analysis, Investigation, Methodology, Visualization

Fourth Author: Data Curation, Formal Analysis, Investigation, Methodology, Visualization

Fifth Author: Conceptualization, Data Curation, Formal Analysis, Investigation, Methodology, Visualization

Sixth Author: Data Curation, Formal Analysis, Investigation, Methodology, Visualization

Seventh Author: Data Curation, Formal Analysis, Investigation, Methodology, Visualization

Eighth Author: Data Curation, Investigation

Ninth Author: Data Curation, Investigation

Tenth Author: Funding Acquisition, Project Administration, Resources

Eleventh Author: Funding Acquisition, Project Administration, Resources

Twelfth Author: Data Curation, Investigation

Thirteenth Author: Data Curation, Investigation

Fourteenth Author: Data Curation, Investigation

Fifteenth Author: Data Curation, Investigation

Sixteenth Author: Data Curation, Investigation

Seventeenth Author: Data Curation, Investigation

\section{Conflict of Interest}

The author(s) declare that they have no known competing financial or non-financial, professional, or personal conflicts that could have appeared to influence the work reported in this paper.

\section{Acknowledgements}

We would like to express our gratitude to the executives and the staff of Çınar Engineering Consulting Co. who executed this project.

\section{References}

Akar, B., \& Şahin, B. (2014). New desmid records of Karagöl Lake in Karagöl-Sahara National Park (Şavşat-
Artvin/Turkey). Turkish Journal of Fisheries and Aquatic Sciences, 14(1), 269-274.

http://dx.doi.org/10.4194/1303-2712-v14_1_29

Anonymous, (2006). EN 15204, European Committee for Standardization. Water quality-Guidance standard on the enumeration of phytoplankton using inverted microscopy (Utermöhl technique).

Apaydın-Yağcı, M., \& Turna, i.i.i. (2002). A new record for the algal flora of Turkey: Chaetomorpha crassa (C.ag.) kütz. (Cladophoraceae, Chlorophyceae). Turkish Journal of Botany, 26, 171-174.

http://dergipark.gov.tr/tbtkbotany/issue/ 11839/ 141429

Atıcı, T. (2002). Nineteen new records from Sarıyar Dam Reservoir phytoplankton for Turkish Freshwater algae. Turkish Journal of Botany, 26, 485-490.

Aysel, V. (2005). Check-List of the Freshwater Algae of Turkey. Journal of the Black Sea/Mediterranean Environment, 11(1): 1-124.

Aysel, V., Dural, B., \& Gezerler-Şipal, U. (1993). Two new records of Cyanophyceae for the Algal Flora of Turkey. Turkish Journal of Botany, 17, 263-266.

Baker P.D., \& Fabbro L.D. (2002). A guide to identification of common blue-green algae (cyanoprokaryotes) in Australian freshwaters. Co-operative Research Centre for Freshwater Ecology Identification, Vol.25, Thurgoona, Australia.

Baykal, T., Akbulut, T., Açıkgöz, İ., Udoh, A.U., Yıldız, K., \& Şen, B. (2009). New Records for the Freshwater Algae of Turkey. Turkish Journal of Botany, 33, 141-152.

Baykal, T., Erkaya, I.A., Udoh, A.U., Akbulut, A., Yıldız, K., \& Şen, B. (2012). New records for the freshwater algae of Turkey (Tigris Basin). Turkish Journal of Botany, 36, 747760.

Brummitt, R.K., \& Powell, C.E. (1992). Authors of Plant Names. A List of Authors of Scientific Names of Plants, With Recommended Standard Forms of Their Names, Including Abbreviations. Royal Botanic Gardens, Kew.

Borics, G., Tóthmérész, B., Várbíró, G., Grigorszky, I., Czébely, A., \& Görgényi, J. (2016). Functional phytoplankton distribution in hypertrophic systems across water body size. Hydrobiologia, 764(1), 81-90. https://doi.org/10.1007/s10750-015-2268-3

Compère, P. (1986). Flore pratique des algues d'eau douce de Belgique. Vol.1, Cyanophyceae, Jardin botanique national de Belgique, Meise, Belgique.

Çelekli, A., Kayhan, S., \& Çetin, T. (2020). First assessment of lakes' water quality in Aras River catchment (Turkey); Application of phytoplankton metrics and multivariate approach. Ecological Indicators, 117, 106706. https://doi.org/10.1016/j.ecolind.2020. 106706

Demir, A.N., Fakıoğlu, Ö., \& Dural, B. (2014). Phytoplankton functional groups provide a quality assessment method by the $Q$ assemblage index in Lake Mogan (Turkey). Turkish Journal of Botany, 38(1), 169-179.

Desikachary, T.V. (1959). Cyanophyta. Indian Council of Agricultural Research, New Delhi.

DGWM (Ministry of Agriculture and Forestry, General Directorate of Water Management) (2015a). Türkiye'de Havza Bazında Hassas Alanların ve Su Kalitesi Hedeflerinin Belirlenmesi Projesi. Final Raporu, Cilt-1.

DGWM (Ministry of Agriculture and Forestry, General Directorate of Water Management) (2015b). Yer Üstü Suları, Yer Altı Suları ve Sedimentten Numune Alma ve Biyolojik Örnekleme Tebliği, Resmi Gazete, Sayı: 29274. 
Dillard, G.E. (2000). Freshwater algae of the Southeastern United States: Part 7. Pigmented Euglenophyceae. Bibliotheca Phycologica, Band 106, Berlin, DEU, Cramer.

Ettl, H., \& Gärtner, G. (1988). Süßwasserflora von Mitteleuropa, Bd. 10: Chlorophyta II: Tetrasporales, Chlorococcales, Gloeodendrales. Spektrum Akademischer Verlag, Stuttgart, DEU.

Fenchel, T., \& Finlay, B.J. (2004). The ubiquity of small species: patterns of local and global diversity. BioScience, 54, 777-784.

Foreign Relation Office of DSi (2014). Water and DSI-60 years full of realized projects. Booklet by Ministry of Forestry and Water Affairs. (English).

Geitler, L. (1925). Die Süsswasser-Flora Deutschlands, Österreichs und der Schweiz, Heft 12: Cyanophyceae, Cyanochloricinae = Chlorobacteriaceae. Vol.1, Jena Verlag Gustav Fischer.

Gönülol, A., Öztürk, M., \& Öztürk, M. (1996). A check-list of the freshwater algae of Turkey. Ondokuz Mayıs University, Faculty of Arts and Science, Journal of Science, 7(1), 8-46.

Graneli, E., \& Turner, J.T. (2006). Ecology of Harmful Algae. Ecological Studies, Vol. 189. Springer, Verlag Berlin Heidelberg.

Guiry, M.D., \& Guiry, G.M. (2021). AlgaeBase. World-wide electronic publication. Galway: National University of Ireland. https://www.algaebase.org

Heering, W. (1914). Chlorophyceae III. Ulothrichales, Microsporales, Oedogoniales. In A. Pascher (Ed.), Die Süswasser-Flora Von Deutschlands, Österreich und der Schweiz; Heft 6 (pp. 1-250). Jena Verlag Von Gustav Fischer.

Hillebrand, H. (2004). On the generality of the latitudinal diversity gradient. American Naturalist, 163, 192-211.

Hoşgören, M.Y. (1994). Lakes of Turkey. Turkish Geographical Review, 29, 19-51.

Huber-Pestalozzi, G. (1961). Die Binnengewässer, Band XVI. In A. Thienemann (Ed.), Das Phytoplankton Des Süsswassers, 5. Teil, Chlorophyceae (Grünalgen) Ordnung: Volvocales. (pp. 1-744). E. Schweizerbart'sche Verlagsbuchhandlung (Nagele u. Obermiller), Stuttgart.

Huber-Pestalozzi, G. (1962). Das phytoplankton Des Süsswassers, 1. Teil, Blaualgen, Bakterien. E. Schweizerbarth'sche Verlagsbuchhandlung (Nagele u. Obermiller), Stuttgart.

Huber-Pestalozzi, G. (1969). Das Phytoplankton des Süsswassers, 4. Teil, Euglenophycean. E. Schweizerbarth'sche Verlagsbuchhandlung (Nagele u. Obermiller). Stuttgart.

Huber-Pestalozzi, G. (1972). Die Binnengewässer, Band XVI, In A. Thienemann (Ed.), Das Phytoplankton Des Süsswassers, 6. Teil, Chlorophyceae (Grünalgen), Ordnung: Tetrasporales (pp. 1-116). E. Schweizerbart'sche Verlagsbuchhandlung (Nagele u. Obermiller), Stuttgart.

Huber-Pestalozzi, G. (1983). Das phytoplankton Des Süsswassers, 7. Teil, 1.Halfte, Chlorophyceae (Grünalgen) Ordnung: Chlorococcales. E. Schweizerbarth'sche Verlagsbuchhandlung (Nagele u. Obermiller), Stuttgart.

John, D.M., Whitton, B.A., \& Brook, A.J. (2003). The Freshwater Algal Flora of the British Isles: An Identification Guide to Freshwater and Terrestrial Algae. The Natural History Museum and The British Phycological Society, Cambridge: Cambridge University Press.
Joosten, A.M. (2006). Flora of the Blue-green Algae of the Netherlands: The non- filamentous species of inland waters. KNNV Uitgeverij, Netherlands.

Komárek, J. (2013). Cyanoprokaryota, Vol 19/3: Heterocytous Genera. In B. Bübel, G. Gärtner, L. Krienitz \& M. Schagerl (Eds.), Freshwater Flora of Central Europe (pp. 1-1131). Spektrum Akademischer Verlag, Berlin, Heidelberg.

Komárek J., \& Anagnostidis K. (1999). Cyanoprokaryota, Vol 19/1: Chroococcales. In H. Ettl, J. Gerloff, H. Heynig, D. Mollenhaueur (Eds.), Freshwater Flora of Central Europe (pp. 1-548). Spektrum Akademischer Verlag, Berlin, Heidelberg.

Komárek, J., \& Anagnostidis, K. (2005). Cyanoprokaryota, Vol 19/2: Oscillatoriales. In B. Bübel, G. Gärtner, L. Krienitz \& M. Schagerl (Eds.), Freshwater Flora of Central Europe (pp. 1-759). Spektrum Akademischer Verlag, Berlin, Heidelberg.

Leibold, M.A. (1996). A graphical model of keystone predators in food webs: trophic regulation of abundance, incidence and diversity patterns in communities. American Naturalist, 147, 784-812.

Maileht, K., Nõges, T., Nõges, P., Ott, I., Mischke, U., Carvalho, L., \& Dudley, B. (2013). Water colour, phosphorus and alkalinity are the major determinants of the dominant phytoplankton species in European lakes. Hydrobiologia, 704(1), 115-126.

Maraşlıoğlu, F., Soylu, E.N., \& Gönülol, A. (2005). Seasonal variation of the phytoplankton of Lake Ladik Samsun, Turkey. Journal of Freshwater Ecology, 20(3), 549-553. http://doi.org/10.1080/02705060.2005.9664770

Maraşlıoğlu, F., \& Gönülol, A. (2021). Turkish algae. Turkeywide electronic publication. Çorum, Turkey. http://turkiyealgleri.hitit.edu.tr

Maraşlıoğlu, F., Soylu, E.N., Demir, N., Çelekli, A., Sömek, H., Öterler B., Çetin T., Karaaslan, Y., Sevindik, T.O., Coşkun, T., Solak, C.N., \& Temizel, B. (2021). New Records for The Turkish Freshwater Algal Flora in Twenty Five River Basins of Turkey, Part VI: Charophyta. Trakya University Journal of Natural Sciences, 22(2), 111-129. http://doi.org/10.23902/trkjnat.875740

Morkoyunlu Yüce, A., \& Aktaş, M. (2020). A Study on Algae and Water Qualities of Tahtalı, Davuldere and Çayırköy Ponds (Kocaeli). Journal of the Institute of Science and Technology, 10(3), 1539-1550.

Morin, P.J., \& Fox, J.W. (2004) Diversity in the deep blue sea. Nature, 429, 813-814.

Öterler, B., Elipek, B.C., \& Arat, S.M. (2018). Influence of environmental conditions on the phytoplankton community assemblages in Süloğlu Reservoir (Edirne, Turkey). Turkish Journal of Fisheries and Aquatic Sciences, 18(8), 969-982.

Özer, T.B., Erkaya, I.A., Udoh, A.U., Akbulut, A., Yıldız, K. \& Şen, B. (2012). New records for the freshwater algae of Turkey (Tigris Basin). Turkish Journal of Botany, 36(6), 747-760. http://dx.doi.org/10.3906/bot-1108-16

Öztürk, M., Gönülol, A., \& Öztürk, M. (1995a). Türkiye alg florası için yeni bir kayıt: Pleurotaenium trabecular (Ehr.) ex Nägeli (Desmidiaceae), Ondokuz Mayıs University, Faculty of Arts and Science, Journal of Science, 6(1), 212218.

Öztürk, M., Gezerler-Şipal, U., Güner, H., Gönülol, A., \& Aysel, V. (1995b). Closterium kuetzingii Bréb. var. kuetzingii (Conjugatophyceae, Desmidiales), A new record for the algal flora of Turkey. Ege Journal of Fisheries and Aquatic Sciences, 12(1-2), 145-149. 
Padisák, J., Vasas, G., \& Borics, G. (2016). Phycogeography of freshwater phytoplankton: traditional knowledge and new molecular tools. Hydrobiologia, 764(1), 3-27.

Park, J.G. (2012). Algal Flora of Korea: Cyanophyta: Cyanophyceae: Chroococcales, Oscillatoriales. Freshwater Cyanoprokaryota II, Volume 5, Number 2. National Institute of Biological Resources Environmental Research Complex, Hwangyeong-ro 42, Seo-gu Incheon, 404-708, Republic of Korea.

Philipose, M.T. (1967). Chlorococcales. I.C.A.R. Monographs on Algae, New Delhi.

Phillips, G., Morabito, G., Carvalho, L., Lyche Solheim, A., Skjelbred, B., Moe, J., Andersen, T., Mischke, U., de Hoyos, C., \& Borics, G. (2010). Report of lake phytoplankton composition metrics, including a common metric approach for use in intercalibration by all GIGs. Deliverable D3.1-1. http://www.wiser.eu/results/deliverables/

Ptacnik, R., Andersen, T., Brettum, P., Lepistö, L., \& Willén. E. (2010). Regional species pools control community saturation in lake phytoplankton. Proc Biol Sci., 277(1701), 3755-3764. http://doi.org/10.1098/rspb.2010.1158

Reynolds, C.S. (2006). Ecology of Phytoplankton. Cambridge University Press, Cambridge, UK.

Ricklefs, R.E. (1987). Community diversity: relative roles of and regional processes. Science, 235, 167-171.

Sevindik, T.O., Çelik, K., \& Gönülol, A. (2010). Twenty-four new records for the freshwater algae of Turkey. Turkish Journal of Botany, 34, 249-259.

Sevindik, T.O., Çelik, K., \& Gönülol, A. (2011). Twenty new records for Turkish freshwater algal flora from Çaygören and Ikizcetepeler reservoirs (Balıkesir, Turkey). Turkish Journal of Fisheries and Aquatic Sciences, 11, 399-406. http://doi.org/10.4194/1303-2712-v11_3_09

Sevindik, T.O., Gönülol, A., Önem, B., Tunca, H., \& Arabacı, S. (2015). Thirty new records for Turkish freshwater algal flora from Danamandıra Ponds (Silivri, İstanbul) and North Mollaköy Lake (Sakarya). Biological Diversity and Conservation, 8(2), 4-15.

Sevindik, T.O., Celik, K., \& Naselli-Flores, L. (2017a). Spatial heterogeneity and seasonal succession of phytoplankton functional groups along the vertical gradient in a mesotrophic reservoir. In Annales de LimnologieInternational Journal of Limnology, 53, 129-141.

Sevindik, T.O., Gönülol, A., Tunca, H., Gürsoy, N.Y., Küçükkaya, Ş.N., \& Durgut Kınalı, Z. (2017b). Nineteen new records for Turkish freshwater algal flora from Lake Taşkısığı and Lake Little Akgöl. Biological Diversity and Conservation, 10(1), 69-78.

Smith, V.H., Foster, B.L., Grover, J.P., Holt, R.D., Leibold, M.A., $\&$ deNoyelles. F. (2005). Phytoplankton species richness scales consistently from laboratory microcosms to the world's oceans. Proceedings of the Natural Academy of Sciences of USA, 102(12), 4393-4396. https://doi.org/10.1073/pnas.0500094102

Sommer, U. (1993). Phytoplankton competition in Plußsee: a field test of the resource-ratio hypothesis. Limnology and Oceanography, 38(4), 838-845.
Sömek, H., Balık, S., \& Ustaoğlu, M.R. (2005). Topçam Baraj Gölü (Çine-Aydın) Fitoplanktonu ve Mevsimsel Değişimleri. Süleyman Demirel Üniversitesi Eğirdir Su Ürünleri Fakültesi Dergisi, 1(1), 26-32.

Stomp, M., Huisman, J., Vörös, L., Pick, F.R., Laamanen, M., Haverkamp, T., \& Stal, L.J. (2007). Colourful coexistence of red and green picocyanobacteria in lakes and seas. Ecology Letters, 10(4), 290-298.

Stomp, M., Huisman, J., Mittelbach, G.G., Litchman, E., \& Klausmeier, C.A. (2011). Large-scale biodiversity patterns in freshwater phytoplankton. Ecology, 92(11), 2096-2107.

Şahin, B. (1998). Some new records of desmids from Turkey. Pakistan Journal of Botany, 30(1), 7-13.

Şahin, B. (2000). Some new desmids records for freshwater algal flora of Turkey. Flora Mediterranea, 10, 223-226.

Şahin, B. (2002). Contribution to the desmid flora of Turkey. Algological Studies, 107, 39-48.

Şahin, B. (2005). A preliminary checklist of desmids of Turkey. Cryptogamie, Algologie, 26(4), 399-415.

Şahin, B. (2007). Two New Records for the Freshwater Algae of Turkey. Turkish Journal of Botany, 31, 153-156. http://doi.org/31-2-8-0605-14.

Şahin, B. (2009). Contribution to the desmid flora of Turkey. Turkish Journal of Botany, 33, 457-460. http://doi.org/10.3906/bot-0809-15.

Şahin, B., \& Akar, B. (2007). The desmid flora of some high mountain lakes of the Turkish Doğu Karadeniz region. Pakistan Journal of Botany, 39(5), 1817-1832.

Taşkın, E., Akbulut, A., Yıldız, A., Şahin, B., Şen, B., Uzunöz, C., Solak, C., Başdemir, D., Sevik, F., Sönmez, F., Açkgöz, I., Pabuccu, K., Öztürk, M., Alp, M.T., Albay, M., Çakır, M., Özbay, Ö., Can, Ö., Akçaalan, R., Atıcı, T., ........ Zengin, Z.T. (2019). Turkey algae list. Ali Nihat Gökyiğit Foundation Publication, İstanbul. 804 pp.

Tilman, D., Kilham S.S., \& Kilham, P. (1982). Phytoplankton community ecology: the role of limiting nutrients. Annual Review of Ecology and Systematics, 13, 349-372.

Varol M., \& Fucikova, K. (2015). Four New Records for the Freshwater Algae of Turkey. Journal of Limnology and Freshwater Fisheries Research, 1(2), 83-88. http://dx.doi.org/ 10.17216/ LimnoFish-5000119624

Varol, M., \& Şen, B. (2016). New Records of Euglenophyceae for Turkish Freshwater Algae. Turkish Journal of Fisheries and Aquatic Sciences, 16, 219-225. http://dx.doi.org/ 10.4194/1303-2712-v16_2_01

Wang, J., Soininen, J., Zhang, Y., Wang, B., Jang, X., \& Shen J. (2011). Contrasting patterns in elevational diversity between microorganisms and macroorganisms. Journal of Biogeography, 38, 595-603.

Winslow, L.A., Read, J.S., Hanson, P.C., \& Stanley, E.H. (2015). Does lake size matter? Combining morphology and process modeling to examine the contribution of lake classes to population-scale processes. Inland Waters, 5(1), 7-14. https://doi.org/ 10.5268/IW-5.1.740

Yüce, A.M., \& Ertan, Ö.O. (2014). A new record for the freshwater algae of Turkey. Scientific Research Journal, 2(4), 21-22. 